\title{
CONVEX BODIES AND PERIODIC HOMEOMORPHISMS IN HIIBERT SPACE
}

\author{
BY \\ VICTOR L. KLEE, JR.(1)
}

Introduction. We are going to discuss certain topological properties of infinite-dimensional normed linear spaces, the results being most complete for Hilbert space $\mathfrak{S}$. Let us begin by describing some results and questions of previous authors which are closely related to those of the present paper.

From A. Tychonoff's fixed-point theorem $([31], 1935)\left({ }^{2}\right)$ it follows that in the weak topology, the unit cell $C=\{x \mid\|x\| \leqq 1\}$ of $\mathfrak{S}$ must have the fixedpoint property; that is, every weakly continuous map of $C$ into itself admits at least one fixed point. In the norm topology, on the other hand, S. Kakutani ([13], 1943) described a homeomorphism without fixed points of $C$ onto itself. He used this to show that the unit sphere $S=\{x \mid\|x\|=1\}$ is contractible and is a deformation retract of $C$. At the end of his paper, Kakutani raised several questions. Are any two of $\mathfrak{S}, C$, and $S$ homeomorphic? Does $C$ admit a periodic homeomorphism without fixed points? What is the situation in general Banach spaces? In partial answer to the last question, J. Dugundji ([7], 1951) proved that the unit cell of a normed linear space has the fixed point property only if the space is finite-dimensional. P. A. Smith had proved ([26], 1941) that each prime-period homeomorphism of Euclidean $n$-space $E^{n}$ must have a fixed point and asked ([9, p. 259], 1949) whether $\mathfrak{H}$ admits a period two homeomorphism without fixed points. O. H. Keller ([14], 1931) proved that the infinite-dimensional compact convex subsets of $\mathfrak{S}$ are mutually homeomorphic and all homogeneous. W. A. Blankinship ([3], 1951) showed that if $X \subset \mathfrak{S}$ and $\mathrm{Cl} X$ is compact, then $\mathfrak{S} \sim X$ is contractible.

In the present paper we answer the questions of Kakutani and Smith, strengthen the theorems of Keller and Blankinship, and establish some further topological properties of convex bodies and periodic homeomorphisms in $\mathfrak{S}$. The principal tools employed are (a) Mazur's homeomorphism [22] of the space $\left(L^{1}\right)$ onto the space $\left(L^{2}\right)$; (b) the existence in every nonreflexive normed linear space of a decreasing sequence of bounded closed convex sets with empty intersection; (c) the existence in $\left(L^{1}\right)$ of a one-parameter con-

Presented to the Society, April 28, 1950 and December 28, 1951, and to the International Congress of Mathematicians, September 6, 1950; received by the editors May 30, 1951.

(1) The paper was written at the University of Virginia and reorganized into its present form while the author held a National Research Fellowship at the Institute for Advanced Study.

(2) Numbers in brackets indicate the list of references at the end of the paper. 
tinuously decreasing family of linear sets. (The author is indebted to Dr. Olof Hanner for mentioning (c) in a conversation.)

Organization of the paper is such that designation of various results as lemma, theorem, etc., did not seem appropriate. Thus a simple numbering scheme is employed, with (13.2), for example, denoting the second result of Chapter I, §3. Omission of the Roman numeral in a reference indicates that the result referred to is in the same chapter as the reference.

The results of greatest intrinsic interest are probably those of §II1 and Chapter III. Suppose, for example, that $E$ is either a nonreflexive normed linear space or an infinite-dimensional $l^{p}$-space. Then there is a homeomorphism of period two without fixed points of $E$ onto itself which takes the unit cell onto itself. Every hyperplane in $E$ is homeomorphic with the unit sphere. And if $X$ is an arbitrary compact subset of $E$, then there is an isotopy whose initial transformation is the identity map on $E$ and terminal transformation is a homeomorphism of $E$ onto $E \sim X$.

For Hilbert space $\mathfrak{S}$, these results are considerably sharpened and extended. $\mathfrak{S}$ is homeomorphic with both its unit cell and its unit sphere. If $B$ is a closed convex body in $\mathfrak{S}$, then $B$ is homeomorphic with $\mathfrak{S}$ and its boundary is homeomorphic with either $\mathfrak{S}$ or $\mathfrak{S} \times S^{n}$ for some $n \geqq-1$. If $X$ is an arbitrary finite polytope in $\mathfrak{S}$ and $n \geqq 2$, then $\mathfrak{S}$ admits a homeomorphism of period $n$ with $X$ as its set of fixed points. And for an arbitrary relatively open subset $Y$ of $X, \mathfrak{S}$ admits a homeomorphism of period $n$ whose fixed-point set is homeomorphic with $Y$. For each $\epsilon>0, \mathfrak{S}$ admits a homeomorphism of period $n$ whose fixed-point set is $\{x \mid\|x\| \geqq \epsilon\}$. $\mathfrak{S}$ admits a pointwise-periodic homeomorphism which is not periodic.

Notation. $U$ and $\sim$ will be used for set union and difference, + and - being reserved for the vector operations. The empty set will be denoted by $\Lambda$, and $\phi$ will represent the neutral element of the linear space under consideration. "If and only if" will be rendered by "iff." For distinct points $x$ and $y$ of a linear space, $[x, y]$ will denote the line-segment including its end points, $[x, y[\equiv[x, y] \sim\{y\}$, etc., and Ray $[x y]$ will denote the ray $\{z \mid y \in[x, z]\}$ $\cup[x, y]$. The boundary, interior, closure, $\epsilon$-neighborhood, and convex hull of a set $X$ will be indicated respectively by $F X$, Int $X, \mathrm{Cl} X, N_{\epsilon} X$, and conv $X$. The unit cell and unit sphere of a normed linear space are respectively $\{x \mid\|x\| \leqq 1\}$ and $\{x \mid\|x\|=1\}$. A convex body is a convex set which has an interior point.

We usually avoid the use of parentheses except when their omission would cause confusion. Symbols written consecutively, whether they denote functions or numerical multipliers, are associated to the right; e.g., $f g x$ $\equiv f[g(x)]$.

We shall reduce somewhat the common notational crime of using the subscript $n$ without individual explanations in three entirely different ways: (i) to indicate a specific member of a sequence; (ii) to indicate the sequence it- 
self; (iii) to assert that for all integers $n$, a certain statement is true. We shall use alpha in the second case, beta in the third. Thus $x_{\alpha}$ means the sequence $\left(x_{1}, x_{2}, \cdots\right)$ and $x_{\alpha} \rightarrow x$ means $\lim _{n \rightarrow \infty} x_{n}=x$. The equation $X_{\beta}=Y_{\beta} \cup Z$ means that for each positive (or, occasionally, non-negative) integer $n, X_{n}=Y_{n} \cup Z$. Although this notation still abounds in ambiguities, it is more precise than some and preserves the brevity frequently obtained by writing simply " $x_{n}$ " " $x_{n} \rightarrow x$," and " $X_{n}=Y_{n} \cup Z$ " in the examples above.

\section{Chapter I. The ISOTOPY THEOREM}

I1. Statement of theorem. If $W$ and $Z$ are topological spaces, then a homotopy of $W$ into $Z$ is a continuous function $\eta$ on $W \times[0,1]$ to $Z$. For such a function $\eta, \eta^{*}$ will denote the map of $W \times[0,1]$ into $Z \times[0,1]$ given by $\eta^{*}(w, t)=(\eta(w, t), t)$ and $\eta_{t}$ the map of $W$ into $Z$ given by $\eta_{t} w=\eta(w, t)$ (for $t \in[0,1])$. The homotopy $\eta$ is an isotopy $\langle$ almost-isotopy $\rangle$ iff $\eta_{t}$ is a homeomorphism for each $t \in[0,1]$ (each $t \in] 0,1[\rangle$. The homotopy $\eta$ is an $h$-isotopy $\left\langle\right.$ almost-h-isotopy iff $\eta^{*}$ is a homeomorphism on $W \times[0,1]\langle$ on $W \times] 0,1[\rangle$. The homotopy $\eta$ is onto $Z\langle$ almost-onto $Z\rangle$ iff $\eta_{t} W=Z$ for each $t \in[0,1]$ 〈each $t \in] 0, t[\rangle$. Two mappings $f_{0}$ and $f_{1}$ of $W$ into $Z$ are said to be homotopic iff there is a homotopy $\eta$ of $W$ into $Z$ such that $\eta_{0}=f_{0}$ and $\eta_{1}=f_{1}$. Such adjectives as isotopic, $h$-isotopic, etc., are defined similarly.

The principal tool and theorem of this paper, in terms of which the other results are not so surprising, is the Isotopy Theorem, stated below as

(1.1) Suppose $Y$ is a weakly compact subset of the nonreflexive normed linear space $E$, and $B$ is a bounded convex body in $E$ such that $N_{\delta} Y \subset B$ for some $\delta>0$. Then there is an h-isotopy $\eta$ of $E$ almost-onto $E$ such that (i) if $t=1$ or $x \in E \sim$ Int $B, \eta_{t} x=x$; (ii) $\eta_{0} E=E \sim Y$; (iii) $\lim _{t \rightarrow 0} \eta_{0} \eta_{t}^{-1}=\eta_{1}$ and $\lim _{t \rightarrow 0} \eta_{t} \eta_{0}^{-1}$ $=\eta_{1} \mid E \sim Y$, the convergence being uniform on every compact set.

The present chapter will be devoted to a proof of the Isotopy Theorem.

Notice that (1.1) implies the existence of a homeomorphism of $E$ onto $E \sim Y$ which is the identity on $E \sim$ Int $B$. Such a homeomorphism is the only tool needed in \$II1. To take advantage of this fact and make the results of §II1 more easily accessible, the homeomorphism is obtained in \$I4 as a lemma in the proof of the Isotopy Theorem. Thus, before taking up $\$ 2$, the reader might wish to read the proof of (4.1) and then \$II1.

Before concluding this section we record a remark on isotopies which will later be useful in conjunction with (1.1). Its proof is almost immediate and is left to the reader.

(1.2) Suppose $W$ is a metric space and $\eta$ is an $h$-isotopy of $W$ almost-onto $W$ such that $\eta_{1}$ is the identity map, $\lim _{t \rightarrow 0} \eta_{0} \eta_{t}^{-1}=\eta_{1}$, and $\lim _{t \rightarrow 0} \eta_{t} \eta_{0}^{-1}=\eta_{1} \mid \eta_{0} W$, with convergence uniform on every compact set. Let $\zeta_{t}=\eta_{0} \eta_{t}^{-1}$ for $t>0$ and $\zeta_{0}=\eta_{1}$. Then $\zeta$ is an h-isotopy of $W$ into $W$, with $\zeta_{0} W=W$ and $\zeta_{t} W=\eta_{0} W$ for $t>0$.

I2. Polar nets. In this section we introduce a sort of generalized polar 
coordinate system which will be one of our principal tools. Let $E$ be a normed linear space and $\Sigma$ its unit sphere $\{\sigma \mid\|\sigma\|=1\}$. A polar net is a system $\{(P t, p t) \mid t \in] 0,1]\}$ which satisfies the following conditions (for each $r$ and $s$ in $] 0,1])$ :

(i) $\operatorname{Pr}$ is a bounded closed subset of $E$ which is star-shaped from the point pr;

(ii) $\mathrm{pr} \in \mathrm{Int} \mathrm{Pr}$ and each ray emanating from pr intersects the boundary $\mathrm{FPr}$ exactly once;

(iii) $\operatorname{Pr} \subset$ Int $P s$ whenever $r<s$;

(iv) $\bigcup_{t \in] 0,1]} F P t=P 1 \sim \bigcap_{t \in] 0,1]} P t$;

(v) $p t \mid t \in] 0,1]$ is continuous.

(These conditions are somewhat redundant, but this is not important.)

The role of alpha will be taken, relative to ]0, 1], by tau. Thus (v), for example, could be written " $p \tau$ is continuous."

The set $\cap P \tau$ is called the origin of the polar net. We can define a biunique mapping $T$ of $P 1 \sim \cap P \tau$ onto ]0,1] $\times \Sigma$ by

(1) $T x=(t, \sigma)$ iff $x \in F P t$ and $\sigma=\|x-p t\|^{-1}(x-p t)$, or equivalently,

(2) $T x=(t, \sigma)$ iff $x=p t+\left[\mu_{t}(p t+\sigma)\right]^{-1} \sigma$, where $\mu_{t}$ is the gauge function of $P t$ relative to $p t$. (That is, $\mu_{t} x=[\sup \{m \mid p t+m(x-p t) \in P t\}]^{-1}$.) It will be proved in this section that $T$ is a homeomorphism.

(2.1) If (i) is satisfied, then (ii) is equivalent to

(vi) the gauge function $\mu_{r}$ is continuous;

Also [(iii) and (iv)] is equivalent to

(vii) $\bigcap_{r>_{s}} \operatorname{Pr}=P s$ (whenever $\left.s \in\right] 0,1\left[\right.$ ) and $U_{r<s} P r=I n t P s$ (whenever $s \in] 0,1])$

and implies

(viii) $F P \tau$ is a continuous set-valued function (i.e., if $t_{\alpha} \rightarrow t$, then $\lim F P t_{\alpha}$ $=F P t$.

Proof. If (ii), holds then $\operatorname{FPr}=\left\{x \mid \mu_{r} x=1\right\}$ and Int $\operatorname{Pr}=\left\{x \mid \mu_{r} x<1\right\}$. But then if $0<a<b$,

$$
\left\{x \mid \mu_{r} x \in\right] a, b[\}=p r+[b(\operatorname{Pr}-p r) \sim b(F P r-p r) \sim a(\operatorname{Pr}-p r)],
$$

which is open. And

$$
\left\{x \mid \mu_{r} x \in[0, b[\}=p r+[b(P r-p r) \sim b(F P r-p r)],\right.
$$

which is open. Hence $\mu_{r}$ is continuous and (ii) implies (vi).

Suppose conversely that $\mu_{r}$ is continuous and let $x \in F P r$. Then $x$ is an accumulation point of the set, $E \sim P r$, on which $\mu_{r}>1$, and hence by continuity $\mu_{r} x \geqq 1$. Also (since $\left.x \in P r\right) \mu_{r} x \leqq 1$, so $\mu_{r} x=1$. But then $p r+\lambda(x-p r)$ $\notin \operatorname{Pr}$ when $\lambda>1$, and from this fact (true for all $x \in F P r$ ) condition (ii) follows at once.

Now suppose [(iii) and (iv)] holds and let the first and second statements in (vii) be denoted by (vii') and (vii") respectively. From (iii) it follows at once that $n_{r>d} \operatorname{Pr} \supset P s$ and $U_{r<e} \operatorname{Pr} C$ Int $P s$. We wish to show also that 
$\bigcap_{r>s} \operatorname{Pr} \supset P s$ and Int $P s \subset \bigcup_{r<s} P r$. Suppose $x \in \cap_{r>s} P r$. By (iv) we have either $x \in \cap P \tau \subset P s$ or $x \in F P_{t}$ for some $t$. But $u>s$ implies (via (iii)) $x \in$ Int $P u$, so we have $t \leqq s$ and $x \in P s$. Hence (vii') holds. And if $y \in$ Int $P s$, then (by (iv)) either $y \in \cap P \tau$ or $y \in F P t$ for some $t$, and from (iii) it follows that $t<s$. Hence (vii') holds and we have shown that [(iii) and (iv)] implies (vii).

Clearly (vii") implies (iii), and this in turn implies that $U F P \tau \subset P 1$ $\sim \cap P \tau$. (This is one of the redundancies in conditions (i)-(iv).) Now suppose $x \in P 1 \sim \cap P \tau$ and let $s=\inf \{r \mid x \in P r\}$. Since $s>0$, (vii') implies $x \in P s$. And if $x \in$ Int $P s$, (vii") implies $x \in P t$ for some $t<s$, which contradicts the definition of $s$. Hence $x \in F P s$ and (iv) holds. We have shown that (vii) implies [(iii) and (iv)], and hence that they are equivalent.

We show finally that (vii) implies (viii). Consider a sequence $t_{\alpha}$ of numbers in $] 0,1]$ with $\left.\left.t_{\alpha} \rightarrow t \in\right] 0,1\right]$. We wish to show that $\lim F P t_{\alpha}=F P t$. Let $x \in \lim \sup F P t_{\alpha}$. Then there is a subsequence $r_{\alpha}$ of $t_{\alpha}$ and a sequence of points $x_{\alpha} \rightarrow x$ with $x_{\beta} \in F P r_{\beta}$. Since $x \notin P t / 2$ we have $x \in F P u$ for some $\left.\left.u \in\right] 0,1\right]$, and wish to show that $u=t$. If some infinite subsequence of $r_{\alpha}$ is monotone decreasing, it follows from (vii') that $x \in P t$. If some subsequence is monotone increasing, the same conclusion follows from (vii' ${ }^{\prime \prime}$. Thus $u \leqq t$. Now if $u<t$, it follows from (vii ${ }^{\prime \prime}$ ) that $x \in \operatorname{Int} P v$ for some $v \in$ ] $u$ [, which is clearly impossible. Hence $u=t$ and we have proved that lim sup $F P t_{\alpha} \subset F P t$. Thus $F P \tau$ is upper semi-continuous.

Now suppose $t_{\alpha} \rightarrow t$ as above and $x \in F P t$. We consider only the case $t<1$ since the other is similar. Let $U$ be an arbitrary connected open set containing $x$. It follows from (vii) that $U$ contains points of $F P r$ for some $r<t$ and of $F P s$ for some $s>t$. But (iii) implies that if $b \in] a, c[C] 0,1]$, then $F P b$ separates $F P a$ from $F P c$ in $E$. Thus since $U$ is connected it must contain points of $F P u$ for each $u \in[r, s]$. Hence there are points $x_{\beta} \in F P t_{\beta}$ such that $x_{\alpha} \rightarrow x$. We then have $F P t \subset \lim$ inf $F P t_{\alpha}$, so $F P \tau$ is also lower semi-continuous and the proof of (2.1) is complete.

Notice that condition (i) was employed only in proving the equivalence of (ii) with (vi), that [(iii) and (iv)] is equivalent to (vii) in every topological space, and implies (viii) in every locally connected metric space.

(2.2) Suppose $(P \tau, p \tau)$ is a polar net having origin $Y$, and the transformation $T$ is defined by (1) above. Then $T$ is a homeomorphism of $P 1 \sim Y$ onto ]0, 1] $\times \Sigma$.

Proof. We show first that $T$ is continuous. Suppose $x_{\alpha} \rightarrow x_{0}$ with $T x_{\beta}$ $=\left(t_{\beta}, \sigma_{\beta}\right)$ and let $t_{n \alpha}$ be an arbitrary convergent subsequence of $t_{\alpha}-$ say $t_{n \alpha} \rightarrow t$. Note first that $t \neq 0$. For suppose $t=0$ and consider an arbitrary $r \in] 0,1]$. Since $t_{n \alpha} \rightarrow 0$ we have $x_{n j} \in \bigcap_{s \in 10, r]} P s$ for all sufficiently large $j$, and hence $x_{0} \in \bigcap_{s \in 10, r]} P s$. But then $x_{0} \in \cap P \tau$, a contradiction since $T$ is not defined on $\cap P \tau$. Now since $t>0$ and $x_{n \beta} \in F P t_{n \beta}$, it follows from (viii) that $x_{0} \in F P t$, whence $t=t_{0}>0$. But then $t_{\alpha} \rightarrow t_{0}$ and from (v) it follows that $p t_{\alpha}$ $\rightarrow p t_{0}$. Now we have $\sigma_{\beta}=\left\|x_{\beta}-p t_{\beta}\right\|^{-1}\left(x_{\beta}-p t_{\beta}\right)$ and $x_{0} \neq p t_{0}$. Hence $\sigma_{\alpha} \rightarrow \sigma_{0}$ and 
thus $T$ is continuous.

Now suppose on the other hand that $t_{\alpha} \rightarrow t_{0}$ and $\sigma_{\alpha} \rightarrow \sigma_{0}$ with $T x_{\beta}=\left(t_{\beta}, \sigma_{\beta}\right)$. Since $x_{\beta}=p t_{\beta}+\sigma_{\beta}\left\|x_{\beta}-p t_{\beta}\right\|$ we have

$$
\frac{x_{\beta}-p t_{0}}{\left\|x_{\beta}-p t_{0}\right\|}=\frac{\left\|x_{\beta}-p t_{\beta}\right\|}{\left\|x_{\beta}-p t_{0}\right\|}\left(\sigma_{\beta}+\frac{p t_{\beta}-p t_{0}}{\left\|x_{\beta}-p t_{\beta}\right\|}\right),
$$

and the sequences $\left\|x_{\alpha}-p t_{0}\right\|$ and $\left\|x_{\alpha}-p t_{\alpha}\right\|$ are bounded away from zero, so

$$
\lim \left\|x_{\alpha}-p t_{0}\right\|^{-1}\left(x_{\alpha}-p t_{0}\right)=\lim \sigma_{\alpha}=\sigma_{0}=\left\|x_{0}-p t_{0}\right\|^{-1}\left(x_{0}-p t_{0}\right) .
$$

Now (viii) implies that the limit of every convergent subsequence of $x_{\alpha}$ must be in $F P t_{0}$. And since by (ii) the ray Ray [pt $t_{0}, x_{0}$ [ intersects $F P t_{0}$ only once, we must have $x_{\alpha} \rightarrow x_{0}$ and the proof is complete.

I3. Admissible triples. To prove the Isotopy Theorem, we establish in $\$ 4$ the existence of a one-parameter family $\mathfrak{B}^{r}=\left(P^{r} \tau, p \tau\right)$ of polar nets having certain useful properties. In doing this, we first define the sets $P^{r} t$ for discrete values of $r$ and $t$ and then fill in the gaps. In order to describe the filling-in process employed, we introduce here the notion of an admissible triple.

For a normed linear space $E$ (which is arbitrary but will remain fixed throughout this section), an admissible triple is an ordered triple $(G, K, J)$ where $G$ is open and convex, $G \subset K \subset J \subset E$, and both $K$ and $J$ are bounded, closed, and star-shaped from $G$. Let $\mathfrak{T}$ denote the class of all admissible triples and consider an arbitrary triple $(G, K, J) \in \mathfrak{T}$. For each ray $\rho$ emanating from $G$, let $\rho_{0}=\rho \cap F K$ and $\rho_{1}=\rho \cap F J$. (Each of these is a single point.) For each $r \in[0,1]$ let

$$
(G, K, J, r)=K \cup\left\{\rho_{0}+s\left(\rho_{1}-\rho_{0}\right) \mid 0 \leqq s<r, \rho \text { emanating from } G\right\} .
$$

The closure of $(G, K, J, r)$ will be denoted by $[G, K, J, r]$.

(3.1) Suppose $(G, K, J) \in \mathfrak{T}$. Then $[G, K, J, 0]=K$ and $[G, K, J, 1]=J$.

(3.2) Suppose $(G, K, J) \in \mathfrak{T}$ and $r \in[0,1]$. Then $[G, K, J, r]$ is star-shaped from $G$.

Proof. It suffices to prove that $(G, K, J, r)$ is star-shaped from $G$. Suppose, then, that $g \in G, p \in(G, K, J, r)$, and $q \in] g, p[$. We wish to show that $q \in(G, K, J, r)$. If $q \in K$ this is obvious, so suppose $q \notin K$. Then $p \notin K$ and there is a ray $\rho$ emanating from a point $g^{\prime}$ of $G$ such that $p=\rho_{0}+s\left(\rho_{1}-\rho_{0}\right)$ for some $s<r$. Let $g^{\prime \prime} \in\left[g, g^{\prime}\right] \cap \operatorname{Ray}\left[\rho_{1}, q\left[, \rho^{\prime}=\operatorname{Ray}\left[g^{\prime \prime}, q\left[\right.\right.\right.\right.$, and $x=\rho^{\prime} \cap\left[g, \rho_{0}\right]$. Since $\left[g, \rho_{0}\right] \subset K$, we have $\rho_{0}^{\prime} \in[x, q]$. Also, $\rho_{1}^{\prime}=\rho_{1}$, so by a little vector algebra it follows that

$$
\frac{\left\|q-\rho_{0}^{\prime}\right\|}{\left\|\rho_{1}^{\prime}-\rho_{0}^{\prime}\right\|} \leqq \frac{\|q-x\|}{\left\|\rho_{1}-\rho_{0}^{\prime}\right\|} \leqq \frac{\left\|p-\rho_{0}\right\|}{\left\|\rho_{1}-\rho_{0}\right\|}=s<r .
$$

Hence $q \in(G, K, J, r)$ and (3.2) has been proved.

"WCCZ" will mean that $N_{\delta} W \subset Z$ for some $\delta>0$. 
(3.3) Suppose $(G, K, J)$ and $\left(G^{\prime}, K^{\prime}, J^{\prime}\right)$ are admissible triples, with $G \subset \subset G^{\prime}, K \subset \subset K^{\prime}$, and $J \subset J^{\prime}$. Then for each $r \in[0,1[,[G, K, J, r]$ $\subset \subset\left[G^{\prime}, K^{\prime}, J^{\prime}, r\right]$.

Proof. By hypothesis there is an $\epsilon>0$ such that $N_{\epsilon} G \subset G^{\prime}$ and $N_{\epsilon} K \subset K^{\prime}$. We shall show that if $D=\max (\epsilon$, diam. $J)$ and $\delta=\epsilon^{2}(1-r) / D$, then $N_{\delta}(G, K, J, r) \subset\left(G^{\prime}, K^{\prime}, J^{\prime}, r\right)$. Consider an arbitrary $p \in(G, K, J, r)$. If $p \in K$ then we have $N_{\delta} p \subset N_{\epsilon} p \subset K^{\prime} \subset\left(G^{\prime}, K^{\prime}, J^{\prime}, r\right)$. If $p \notin K$, then there is a number $s<r$ and a ray $\rho$ emanating from a point $g$ of $G$ such that $p=\rho_{0}+s\left(\rho_{1}-\rho_{0}\right)$. For the sake of notational simplicity we assume that $\rho_{0}=\phi$. Let $\rho_{0}^{\prime}=\rho$ $\cap F K^{\prime}$. Then $\rho_{0}^{\prime}+s\left(\rho_{1}-\rho_{0}^{\prime}\right)=y \in G^{\prime}$. But $\rho_{0}^{\prime}=k \rho_{1} /\left\|\rho_{1}\right\|$ with $k \geqq \epsilon$, so we have $y=t \rho_{1}$ with $t=s+\left[(1-s) k /\left\|\rho_{1}\right\|\right]$. Now by (3.2) the star from $y$ over $G^{\prime}$ is in $\left(G^{\prime}, K^{\prime}, J^{\prime}, r\right)$, and hence so is the star from $y$ over $N_{\epsilon} g$. This star contains $N_{m \in} p$, where $m=\|y-p\| /\|y-g\|$. But $\|y-g\|<D$ and

$$
\|y-p\|=\left\|\rho_{1}\right\|(t-s)=\left\|\rho_{1}\right\| \frac{(1-s) k}{\left\|\rho_{1}\right\|}>(1-r),
$$

so $m>\epsilon(1-r) / D$ and the proof is complete.

By the Hausdorff distance $h(X, Y)$ between two subsets of $E$ is meant inf $\left\{\epsilon \mid X \subset N_{\epsilon} Y\right.$ and $\left.Y \subset N_{\epsilon} X\right\}$. As thus defined, $h$ is a distance-function for the class of all bounded closed subsets of $E$ and also for the class of all bounded open convex subsets of $E$ (since a bounded open convex set is completely determined once its closure is given). Thus the class $\mathfrak{T}$ of admissible triples is metrized by the function $m$ defined as follows:

$$
m\left((G, K, J),\left(G^{\prime}, K^{\prime}, J^{\prime}\right)\right)=\max \left(h\left(G, G^{\prime}\right), h\left(K, K^{\prime}\right), h\left(J, J^{\prime}\right)\right) .
$$

For each pair of positive numbers $d$ and $D$ such that $d<D$, let $\mathfrak{T}(d, D)$ be the class of all triples $(G, K, J) \in \mathfrak{T}$ such that $\left(N_{d} G, K, J\right) \in \mathfrak{T}$ and diam. $J$ $<D$.

(3.4) The function $[G, K, J, r] \mid(G, K, J) \in \mathfrak{T}(d, D), r \in[0,1]$ is uniformly continuous. That is, to each $\epsilon>0$ corresponds $\delta>0$ such that if $(G, K, J)$ and $\left(G^{\prime}, K^{\prime}, J^{\prime}\right)$ are in $\mathfrak{T}(d, D), r$ and $r^{\prime}$ are in $[0,1], m\left[(G, K, J),\left(G^{\prime}, K^{\prime}, J^{\prime}\right)\right]$ $<\delta$, and $\left|r-r^{\prime}\right|<\delta$, then $h\left([G, K, J, r)\left[G^{\prime}, K^{\prime}, J^{\prime}, r^{\prime}\right]\right)<\epsilon$.

Proof. The following statement is easy to verify:

(i) If $(G, K, J) \in \mathfrak{T}(d, D)$, then $h\left([G, K, J, r],\left[G, K, J, r^{\prime}\right]\right)<\left|r-r^{\prime}\right| D$. We next prove

(ii) If $(G, K, J) \in \mathfrak{T}(d, D)$ and $c<\epsilon d / D$, then $\left[G, \mathrm{Cl}\left(N_{c} K\right), \mathrm{Cl}\left(N_{c} J\right), r\right]$ $\subset N_{\epsilon}[G, K, J, r]$ (for each $r \in[0,1]$ ).

Consider an arbitrary ray $\rho$ emanating from a point $g$ of $G$, and let $x=\rho \cap F \mathrm{Cl}\left(N_{c} J\right)$. For notational simplicity we assume $g=\phi$. There is a point $y$ with $\|y\|<\epsilon d / D$ such that $x+y \in J$. Since $(G, K, J) \in \mathfrak{I}(d, D), J$ is star-shaped from $-d y /\|y\|$ and hence $J \supset[-d y /\|y\|, x+y]$. But this set intersects $[g, x]=[\phi, x]$ at the point $x^{\prime}=(d /(d+\|y\|)) x$ and we have $\left\|x-x^{\prime}\right\|$ 
$=\|x\|\|y\| /(d+\|y\|)<\epsilon$. And $\rho \cap F J \subset\left[x^{\prime}, x[\right.$, so the distance between $\rho$ $\cap F \mathrm{Cl}\left(N_{c} J\right)$ and $\rho \cap F J$ is less than $\epsilon$ for each ray $\rho$ emanating from $G$. By the same argument, this is true of $K$ also, and this yields the desired conclusion.

(iii) If $(G, K, J) \in \mathfrak{T},\left(N_{c} G, K, J\right) \in \mathfrak{T}(d, D)$, and $c<\epsilon d \min ((d+D))^{-1}$, $\left.d\left(d^{2}+D\right)^{-1}\right)$, then $\left[N_{c} G, K, J, r\right] \subset N_{\epsilon}[G, K, J, r]$.

Consider an arbitrary ray $\rho^{\prime}$ emanating from a point $g^{\prime}$ of $N_{c} G$. Let $g \in N_{c} g^{\prime} \cap G$ and $\rho=$ Ray [ $g, \rho_{1}^{\prime}[$ [. To establish (iii) it will suffice to show that $\left\|\rho_{0}-\rho_{0}^{\prime}\right\|<\epsilon$. For notational simplicity we assume that $g^{\prime}=\phi$. Now let $u=-d g /\|g\|$ and $v=g-u$. Then $u$ and $v$ are both in $N_{d} N_{c} G$, so from one of our hypotheses it follows that $K$ is star-shaped from both $u$ and $v$. Let $p=\operatorname{Ray}\left[v, \rho_{0}^{\prime}\left[\cap \rho\right.\right.$ and $q=\operatorname{Ray}\left[u, \rho_{0}^{\prime}\left[\cap \rho\right.\right.$. Since $\left[v, \rho_{0}^{\prime}\right] \subset K, \rho_{0} \in\left[p, \rho_{1}^{\prime}\right]$. And if $K$ were to intersect ] $\left.q, \rho_{1}^{\prime}\right]$, then it would intersect $\left[\rho_{0}^{\prime}, \rho_{1}^{\prime}[\right.$, which is impossible. Thus $\rho_{0} \in[p, q]$, and proof of (iii) will be completed by showing that $\left\|\rho_{0}^{\prime}-p\right\|<\epsilon$ and $\left\|\rho_{0}^{\prime}-q\right\|<\epsilon$.

For some $t>1, \rho_{1}^{\prime}=t \rho_{0}^{\prime}$. And by a little vector-algebra it can be verified that $q=(1-\mu) g+\mu t \rho_{0}^{\prime}$ and $p=(1-\lambda) g+\lambda t \rho_{0}^{\prime}$, where $\mu=(\|g\|+d) /(t d+\|g\|)$ and $\lambda=d /[t d+(t-1)\|g\|]$. But then

$$
1-\mu t=\frac{\|g\|}{d} \frac{1}{d}-\frac{1}{d t}<\frac{c}{d^{2}}
$$

and

$$
1-\lambda t=\frac{(t-1)\|g\|}{t d+(t-1)\|g\|}<\frac{(t-1)\|g\|}{t d}<\frac{c}{d} .
$$

The inequality on $c$ given in the hypothesis implies that $c(1+D / d)<\epsilon$ and $c\left(1+D / d^{2}\right)<\epsilon$, which gives the desired conclusion since $\left\|\rho_{0}^{\prime}\right\|<D$.

From (i)-(iii) above it can be verified that for $\delta$ sufficiently small we have (with $m\left((G, K, J),\left(G^{\prime}, K^{\prime}, J^{\prime}\right)\right)<\delta$ and $\left|r-r^{\prime}\right|<\delta$ )

$$
\begin{aligned}
& {\left[G^{\prime}, K^{\prime}, J^{\prime}, r^{\prime}\right] } \subset\left[N_{\delta} G, \mathrm{Cl}\left(N_{\delta} K\right), \mathrm{Cl}\left(N_{\delta} J\right), r^{\prime}\right] \subset N_{\epsilon}\left[N_{\delta} G, K, J, r^{\prime}\right] \\
& \subset N_{2 \epsilon}\left[G, K, J, r^{\prime}\right] \subset N_{3 \epsilon}[G, K, J, r],
\end{aligned}
$$

and similarly $[G, K, J, r] \subset N_{3 \epsilon}\left[G^{\prime}, K^{\prime}, J^{\prime}, r^{\prime}\right]$. This completes the proof of (3.4).

It can be verified that if $X$ is bounded, $Y$ is star-shaped, and $F X \subset N_{\epsilon} F Y$, then $X \subset N_{\epsilon} Y$. Thus if $X$ and $Y$ are both bounded and star-shaped, $h(X, Y)$ $\leqq h(F X, F Y)$.

(3.5) Suppose $X$ and $Y$ are both star-shaped from $N_{c} \phi$, both of diameter $<D$, and $h(X, Y)<c / 2$. Then $h(F X, F Y)<2 D c^{-1} h(X, Y)$.

Proof. We assume without loss of generality that $X$ and $Y$ are both closed and hence both star-shaped from $\mathrm{Cl}\left(N_{c} \phi\right)$. Let $\epsilon$ be an arbitrary number such that $h(X, Y)<\epsilon<c$. We shall show that $F X \subset N_{2 D \sigma_{-1 \epsilon}} F Y$. Since $X$ and 
$Y$ enter symmetrically in the hypotheses of (3.5), this yields the desired conclusion.

Consider an arbitrary point $x \in F X$ and let $K$ be the set of all points $u$ such that $x \in$ ] $v, u$ [ for some $v \in N_{c} \phi$. If such a point $u$ were in $\dot{X}$, then from the fact that $X$ is star-shaped from $N_{c} \phi$ it would follow that $x \in \operatorname{Int} X$, which contradicts the fact that $x \in F X$. Thus $K \cap X=\Lambda$. Now by hypothesis there is a point $y^{\prime} \in Y$ such that $\left\|y^{\prime}-x\right\|<\epsilon$. But $Y$ is star-shaped from $\phi$, so $\left[\phi, y^{\prime}\right] \subset Y$, and since $\left\|x^{\prime}-\phi\right\| \geqq c>\epsilon$, there is a point $y \in Y$ such that $\|y-x\|=\epsilon$. Let $z=c \epsilon^{-1}(y-x)$. Then Ray $[z, y[$ intersects Ray $[\phi, x[$ at the point $p=x\left(1+\epsilon(c-\epsilon)^{-1}\|x\|\right)$. And $p \notin Y$, for we have $N_{c \epsilon(c-\epsilon)^{-1}} p \subset K, K \cap X=\Lambda$, and $h(X, Y)<\epsilon<c \epsilon(c-\epsilon)^{-1}$. But $[z, y] \subset Y$, so $F Y$ must intersect $[y, p]$. Now $\|y-x\|=\epsilon\left\langle D c^{-1} \epsilon\right.$ and $\|p-x\|=\|x\| \epsilon(c-\epsilon)^{-1}<2 D c^{-1} \epsilon$, so $x \in N_{2 D c_{-1}^{-1}} F Y$. Hence (since $x$ was an arbitrary point of $F X) F X \subset N_{2 D c}{ }^{-1} \epsilon_{\epsilon} F Y$ and the proof is complete.

For each triple $(c, d, D)$ of positive numbers such that $c+d<D$, let $\mathfrak{T}(c, d, D)$ be the class of all triples $(G, K, J) \in \mathfrak{T}(d, D)$ such that $N_{c} p \subset G$ for some $p$. Then from (3.4) and (3.5) we have

(3.6) The function $F[G, K, J, r] \mid(G, K, J) \in \mathfrak{T}(c, d, D), r \in[0,1]$ is uniformly continuous.

I4. Proof of the theorem. We first establish a relatively easy result (promised in §I1) from which the results of $\S I I 1$ can be derived. (" $W \subset \subset Z$ " will mean that $N_{\delta} W \subset Z$ for some $\delta>0$.)

(4.1) Suppose $Y$ is a weakly compact subset of the nonreflexive normed linear space $E$ and $B$ is a bounded closed convex body in $E$ such that $Y \subset \subset B$. Then there is a homeomorphism of $B$ onto $B \sim Y$ which is the identity on $F B$.

Proof. According to a result of Eberlein [8], $E$ is reflexive iff its unit cell is sequentially compact in the weak topology, and by a theorem of Smulian [28] (a proof contained also in [16]) this in turn is equivalent to the nonexistence of a decreasing sequence of bounded closed convex sets with empty intersection. Thus, since in the present case $E$ is nonreflexive, there are a sequence $C_{0} \supset C_{1} \supset \ldots$ of closed convex sets and an $\epsilon>0$ such that $N_{3 \epsilon} Y$ $\subset B, N_{2 \epsilon} C_{0} \subset B$, and $\cap C_{\alpha}=\Lambda$. For $\beta=1,2, \cdots$, let $G_{\beta}=N_{2-\beta_{\epsilon}} C_{\beta}, H_{\beta}=N_{2-\beta_{\epsilon}} Y$, and let $V_{\beta}$ be the union of all segments $[x, y]$ for which $x \in G_{\beta-1}$ and $y \in H_{\beta}$, replacing $G_{0}$ by $N_{\epsilon} C_{0}$ in the case of $V_{1}$. Let $G_{0}=V_{0}=B$. It is easily verified that $V_{\beta+1} \subset \subset V_{\beta}, G_{\beta+1} \subset \subset G_{\beta} \cap V_{\beta}$, and both $G_{\beta}$ and $V_{\beta}$ are star-shaped from $G_{\beta}$.

We show now that $\cap V_{\alpha}=Y$. Since clearly $\cap V_{\alpha} \supset Y$, we must establish the reverse inclusion. Suppose $q \in \cap V_{\alpha}$. Then there are points $x_{\beta} \in G_{\beta-1}, y_{\beta} \in H_{\beta}$, and numbers $t_{\beta} \in[0,1]$ such that $q=\left(1-t_{\beta}\right) x_{\beta}+t_{\beta} y_{\beta}$. Since $[0,1]$ is compact and $Y$ is weakly compact we can in fact find such sequences for which $t_{\alpha}$ converges (say to $t \in[0,1]$ ) and $Y_{\alpha}$ converges weakly (say to $y \in Y$ ). Now if $t \neq 1$ this implies that $x_{\alpha}$ converges weakly to $x=(1-t)^{-1}(q-t y)$. But each $C_{i}$, being closed and convex, is weakly closed, so we have $x \in \cap C_{\alpha}$, which is im- 
possible. Hence $t=1$ and $q=y \in Y$. Thus $Y=\cap C_{\alpha}$.

We now proceed to define the desired homeomorphism $T$. First pick points $w_{\beta} \in G_{\beta+1}$. Then for each $v \in F V_{\beta}\left\langle u \in F G_{\beta}\right\rangle$ there is a unique point $v^{\prime}\left\langle u^{\prime}\right\rangle$ such that Ray $\left[w_{\beta}, v\left[\cap\left(\mathrm{Cl}\left|V_{\beta}\right| \sim \operatorname{Int} V_{\beta+1}\right)=\left[v^{\prime}, v\right]\left\langle\operatorname{Ray}\left[w_{\beta}, u\right]\right.\right.\right.$ $\cap\left(\mathrm{Cl} G_{\beta} \sim\right.$ Int $\left.\left.G_{\beta+1}\right)=\left[u^{\prime}, u\right]\right\rangle$. Now let $T$ be the identity map on $F B$. And having defined $T$ mapping $F G_{\beta}$ topologically onto $F V_{\beta}$, define $T$ on $\mathrm{Cl} G_{\beta}$ $\sim$ Int $G_{\beta+1}$ and onto $\mathrm{Cl} V_{\beta} \sim$ Int $V_{\beta+1}$ as follows: for each $u \in F G_{\beta}$, let $T$ map $\left[u^{\prime}, u\right]$ linearly onto $\left[v^{\prime}, v\right]$. Then at the $\beta$ th stage, $T$ is a homeomorphism of $\mathrm{Cl} G_{\beta-1} \sim$ Int $G_{\beta}$ onto $\mathrm{Cl} V_{\beta-1} \sim$ Int $V_{\beta}$. Since $\cap G_{\alpha}=\Lambda$ and $\cap V_{\alpha}=Y$, it is clear that $T$ (when defined at all stages) is the desired homeomorphism.

Notice that if $Y$ is a single point, we can simply take $V_{\beta}=N_{2-\beta_{\epsilon}} Y$ and (with a slight modification in the third paragraph) the second paragraph of the proof of (4.1) becomes unnecessary. This case ( $Y$ a single point) is actually all that is needed in $\$ I I 1$.

Some of our principal results could be deduced from (4.2) (a) below. Hence it is of interest to note that it too is equivalent to nonreflexivity.

(4.2) For a normed linear space $E$, the following three statements are equivalent: (a) in $E$ there is a decreasing sequence of bounded closed star-shaped sets whose intersection is empty; (b) in $E$ there is a decreasing sequence of bounded closed convex sets whose intersection is empty; (c) $E$ is nonreflexive.

Proof. Equivalence of (b) and (c) was discussed above, and clearly (b) implies (a). Now suppose (a) holds and let $S_{\alpha}$ be the sequence of star-shaped sets in question. Let $C_{\beta}$ be the set of all points of $S_{\beta}$ from which $S_{\beta}$ is starshaped and $K_{\beta}=\mathrm{Cl}$ conv $\bigcup_{n=\beta}^{\infty} C_{n}$. To show that (a) implies (b) it will suffice to show that $K_{\beta} \subset S_{\beta}$, for then $\cap K_{\alpha}=\Lambda$ and the other requirements are obviously satisfied. To show that $K_{\beta} \subset S_{\beta}$ it suffices to show that for each $k$ and each set of $k$ integers, $n 1 \leqq n 2 \leqq \cdots \leqq n k$, and points $x_{i} \in C_{n i}$, we have conv $\left\{x_{1}, x_{2}, \cdots, x_{k}\right\} \subset S_{n 1}$. For $k=1$, this is obvious. Suppose it has been proved for $k=m-1$ and consider the case $k=m$. By the inductive hypothesis, conv $\left\{x_{2}, \cdots, x_{m}\right\} \subset S_{n 2} \subset S_{n 1}$. But $x_{1} \in C_{n 1}$, so $S_{n 1}$ is star-shaped from $x_{1}$. Thus $S_{n 1}$ contains the star from $x_{1}$ over conv $\left\{x_{2}, \cdots, x_{m}\right\}$, and since this star is precisely conv $\left\{x_{1}, x_{2}, \cdots, x_{m}\right\}$, the proof is complete.

In order to prove the Isotopy Theorem we establish

(4.3) With $Y, B$, and $E$ as in (4.1), there is in $E$ a system of sets $\left\{P^{r} t \mid r\right.$ $\in[0,1], t \in] 0,1]\}$ and of points $\{p t \mid t \in] 0,1]\}$ such that

( $\alpha$ ) for each $r, P^{r} 1=B$;

( $\beta$ ) for each $r,\left(P^{r} \tau, p \tau\right)$ is a polar net $\mathfrak{B}^{r}$;

$(\gamma)$ the origin of $\mathfrak{B}^{0}$ is $Y$, and for $r>0$ the origin of $\mathfrak{B}^{r}$ is $\Lambda$;

( $\delta) F P r t \mid(r, t) \in[0,1] \times] 0,1]$ is continuous in the Hausdorff metric;

(e) if $\mu_{t}^{r}$ is the gauge-function of $P^{r} t$ relative to $p t$, then $\mid \mu_{t}^{r}(p t+\sigma)^{-1}$ $-\mu_{i}^{0}(p t+\sigma)^{-1} \mid<\delta$ whenever $r<\delta$ diam. $\left.\left.B, t \in\right] 0,1\right]$, and $\sigma \in \Sigma$ (the unit sphere of $E$ ).

Proof. It suffices to consider only the case in which $Y$ is nonempty. Let 
$C_{\beta}, G_{\beta}, H_{\beta}$, and $V_{\beta}$ be as in the proof of (4.1). Let $P^{01}=P^{11}=B$ and, for $i \geqq 1, P^{1} 2^{-i}=\mathrm{Cl} G_{i}$ and $P^{0} 2^{-i}=\mathrm{Cl} V_{i}$. Then from the proof of (4.1) we see

(a) $P^{0} 2^{-\beta}$ and $P^{1} 2^{-\beta}$ are both star-shaped from $G_{\beta}$ and $P^{12^{-\beta}} \subset \subset P^{0} 2^{-\beta}$;

(b) $P^{0} 2^{-(\beta+1)} \subset \subset$ Int $P^{0} 2^{-\beta}=V_{\beta}$ and $P^{1} 2^{-(\beta+1)} \subset \subset$ Int $P^{1} 2^{-\beta}=G_{\beta}$.

(c) $\cap P^{1} 2^{-\alpha}=\Lambda$ and $\cap P^{0} 2^{-\alpha}=Y$.

Having defined the sets $P^{r} t$ for certain values of $r$ and $t$, we now proceed to fill in the gaps. And as a start in defining $p \tau$, we first pick $p 2^{-\beta}$ $\in$ Int $P^{1} 2^{-(\beta+3)}$. Now suppose $t=\lambda 2^{-n}+(1-\lambda) 2^{-(n+1)}$, with $\lambda \in[0,1]$. Then let $P^{1} t=\lambda P^{1} 2^{-n}+(1-\lambda) P^{1} 2^{-(n+1)}$ and $p t=\lambda p^{-n}+(1-\lambda) p 2^{-(n+1)}$. From (a) and (b) above and the relevant definitions it follows that the triple involved is in $\mathfrak{I}\left(2^{-(n+1)} \epsilon, 2^{-(n+1)} \epsilon\right.$, diam. $B$ ) (and, in particular, is an admissible triple) so it makes sense to define

$$
P^{0} t=\left[\operatorname{Int} P^{1} 2^{-(n+1)}, P^{0} 2^{-(n+1)}, P^{0} 2^{-n}, \lambda\right] .
$$

By (3.2), $P^{0} t$ is star-shaped from Int $P^{1} 2^{-(n+1)} \supset$ Int $P^{1} t / 4$, so it makes sense to define

$$
P^{r} t=\left[\operatorname{Int} P^{1} t / 4, P^{1} t, P^{0} t, 1-r\right]
$$

for each $r \in\left[0,1\right.$ [. Now clearly $P^{1} \tau$ is continuous, and from (3.4) it follows that $P^{0} \tau$ is continuous. But

$$
\text { (Int } \left.P^{1} t / 4, P^{1} t, P^{0} t\right) \in \mathfrak{T}\left(2^{-(n+3)} \epsilon, 2^{-(n+2)} \epsilon\right. \text {, diam. B), }
$$

so from (3.6) it follows that $F P^{r} t \mid(r, t)$ is continuous. Thus $(\delta)$ holds. $(\alpha)$ follows at once from the fact that $P^{0} 1=P^{11}=B$, so it remains to establish $(\beta),(\gamma)$, and $(\epsilon)$.

To prove ( $\epsilon)$, let us suppose that $r, t$, and $\sigma$ are as indicated and suppose for the sake of notational simplicity that $p t=\phi$. Let $m=\mu_{t}^{r}(p t+\sigma)^{-1}$ and $n=\mu_{i}^{0}(p t+\sigma)^{-1}$. Then $m$ is characterized by the fact that $m \sigma \in F P^{r} t$ and $n$ by the fact that $n \sigma \in F P^{0} t$. But $P^{r} t=\left[\right.$ Int $\left.P^{1} t / 4, P^{1} t, P^{0} t, 1-r\right]$ and $p t$ $\in$ Int $P^{1} t / 4$, so we have $(1-r) n \sigma \in P^{r} t$ and hence $(1-r) n \leqq m \leqq n$. Thus $n-m \leqq n-(1-r) n=r n<\delta$ and $(\epsilon)$ has been proved.

To. establish $(\gamma)$ it suffices to show that $\cap P^{0} 2^{-\alpha}=Y$ (which was done in (c) above) and that $\cap P^{r} 2^{-\alpha}=\Lambda$ when $r>0$. So suppose $q \in \cap P^{r} 2^{-\alpha}$. Then there are points $w_{\beta} \in F P^{1} 2^{-\beta}$ and $y_{\beta} \in F P^{0} 2^{-\beta}$ such that $r w_{\alpha}+(1-r) y_{\alpha} \rightarrow q$. Thus (utilizing the definitions of the sets $P^{0} 2^{-\beta}$ and $P^{1} 2^{-\beta}$, the compactness of $[0,1]$, and the weak compactness of $Y$ ) there are points $x_{\beta} \in G_{\beta}, u_{\beta} \in G_{\beta}$, $v_{\beta} \in H_{\beta}$, and numbers $\lambda_{\beta} \in[0,1]$ such that $\lambda_{\alpha}$ converges (say to $\left.\lambda \in[0,1]\right), v_{\alpha}$ converges weakly (say to $v \in Y$ ), and

$$
r x_{\alpha}+(1-r) \lambda_{\alpha} u_{\alpha}+(1-r)\left(1-\lambda_{\alpha}\right) v_{\alpha} \rightarrow q .
$$

But then if $z_{\beta}=r x_{\beta}+(1-r) \lambda_{\beta} u_{\beta}, z_{\alpha}$ converges weakly to $q-(1-r)(1-\lambda) v$. However, $z_{\beta}=\left[r+(1-r) \lambda_{\beta}\right] g_{\beta}$ with $g_{\beta} \in G_{\beta}$, and since $r+(1-r) \lambda_{\alpha}$ does not $\rightarrow 0$, the weak convergence of $z_{\alpha}$ contradicts the fact that $\cap G_{\alpha}=\Lambda$. 
We wish finally to verify (i)-(v) of $\S 2$ in order to establish $(\beta)$. Since $P^{r} t$ is star-shaped from $P^{1} t / 4$ (by 3.2) and $p t \in \operatorname{Int} P^{1} t / 4$, (i) and (ii) are satisfied. Condition (iii) follows from (3.3) and the relevant definitions, and (v) is obvious. It remains to establish (iv) and we do this only for $P^{r}$ with $r>0$, since the case of $P^{0}$ is similar. Let $x \in B$ and $t=\inf \left\{s \mid x \in P^{r} s\right\}$. Since $\cap P^{r} \tau=\Lambda$, $t>0$, and from the continuity of $P^{r} \tau$ it follows that $x \in P^{r} t$. We wish to show that $x \in F P^{r} t$. Let $q \in \operatorname{Int} P^{1} t / 8$ and notice that for $s>t / 2, P^{r} s$ is star-shaped from $P^{1} t / 8$. Let $p=$ Ray $\left[q, x\left[\cap F P^{r} t\right.\right.$ and let $K$ be the set of all points $v$ such that $x \in] u, v$ [ for some $u \in P^{1} t / 8$. If $x \in F P^{r} t$, then $p \in$ Int $K$ and by continuity of $F P^{r} \tau$ it follows that $F P^{r} s$ intersects $K$ (and hence $x \in P^{r} s$ ) for all $s$ sufficiently close to $t$. Since this contradicts the definition of $t$, we must have $x \in F P^{r} t$. Thus $U F P^{r} \tau=P^{r} 1=B$ and the proof of (4.3) is complete.

For convenient reference, we restate the Isotopy Theorem as

(4.4) Suppose $Y$ is a weakly compact subset of the nonreflexive normed linear space $E$ and $B$ is a bounded convex body in $E$ such that $Y \subset \subset B$. Then there is an h-isotopy $\eta$ of $E$ almost-onto $E$ such that (i) if $t=1$ or $x \in E \sim \operatorname{Int} B$, $\eta_{t} x=x$; (ii) $\eta_{0} E=E \sim Y$; (iii) $\lim _{t \rightarrow 0} \eta_{0} \eta_{t}^{-1}=\eta_{1}$ and $\lim _{t \rightarrow 0} \eta_{t} \eta_{0}^{-1}=n_{1} \mid E \sim Y$, the convergence being uniform on each compact set.

Proof. Let the polar nets $\mathfrak{P}^{r}, 0 \leqq r \leqq 1$, be as in (4.3), and for each $r$ let $T_{r}$ be the transformation of $B \sim \cap P^{r} \tau$ onto $\left.] 0,1\right] \times \Sigma$ which is associated with $\mathfrak{B}^{r}$ by means of equation (1) in $\S I 2$. By (2.2), each $T_{r}$ is a homeomorphism. For each $(x, r) \in B \times[0,1]$, let $\eta(x, r)=T_{r}^{-1} T_{1} x$, and for $(x, r) \in(E \sim B)$ $\times[0,1]$, let $\eta(x, r)=x$. By an argument analogous to that of $(2.2)$, using $(\beta)$ and $(\delta)$ of (4.3), it follows that $\eta^{*}$ is a homeomorphism, and hence (using ( $\alpha)$ and $(\gamma)$ of (4.3)) $\eta$ is an $h$-isotopy of $E$ almost-onto $E$ such that (i) and (ii) are satisfied. To establish (iii), note first that $\eta_{0} \eta_{t}^{-1}=T_{0}^{-1} T_{t}$ and $\eta_{t} \eta_{0}^{-1}$ $=T_{t}^{-1} T_{0}$, so we must show that if $x_{\alpha} \rightarrow y \in Y$ and $r_{\alpha} \rightarrow 0$, then $\left\|T_{0}^{-1} T_{r_{\alpha}} x_{\alpha}-x_{\alpha}\right\|$ $\rightarrow 0$, and that if also $x_{\beta} \in B \sim Y$, then $\left\|T_{\tau_{\alpha}}^{-1} T_{0} x_{\alpha}-x_{\alpha}\right\| \rightarrow 0$. We discuss only the first case, since the second is similar. In this situation, there are numbers $\left.\left.t_{\beta} \in\right] 0,1\right]$ and points $\sigma_{\beta} \in \Sigma$ such that

$$
x_{\beta}=p t_{\beta}+\mu_{t_{\beta}}^{r_{\beta}}\left(p t_{\beta}+\sigma_{\beta}\right)^{-1} \sigma_{\beta}
$$

and

$$
T_{0}^{-1} T_{r_{\beta}} x_{\beta}=p t_{\beta}+\mu_{t_{\beta}}^{0}\left(p t_{\beta}+\sigma_{\beta}\right)^{-1} \sigma_{\beta},
$$

so the desired conclusion follows from $(\epsilon)$ of $(4.3)$.

Chapter II. Topological PROPERTIES OF NONREFlexive AND INFINITE-DIMENSIONAL $L^{p}$-SPACES

II1. Simple properties. Most of the constructions in our proofs will be carried out in nonreflexive spaces. The following lemma makes it possible to apply the results to Hilbert space.

(1.1) Suppose $M$ is a measure-space and $1 \leqq p<\infty$. For each $f \in L^{1} M$, let 
$T f=|f|^{1 / p} \operatorname{sgn} f$. Then $T$ is a homeomorphism of $L^{1} M$ onto $L^{p} M$ which maps the unit cell of $L^{1} M$ onto that of $L^{p} M$. If some point $z$ of $M$ has positive finite measure, then $\left\{f \mid f \in L^{1} M\right.$ and $\left.f z=0\right\}$ is a hyperplane in $L^{1} M$ which maps under $T$ onto a hyperplane in $L^{p} M$. If $M$ contains an infinite sequence $X_{\alpha}$ of pairwise disjoint sets, each of positive finite measure, then $L^{1} M$ is nonreflexive.

Proof. That $T$ is a homeomorphism of $L^{1} M$ onto $L^{p} M$ was proved by Mazur [22] for $M=$ ]0, 1 [, and the same proof is valid for arbitrary $M$. The statements about unit cells and hyperplanes are obvious. To see that $L^{1} M$ is nonreflexive let $f_{\beta}=\left(m_{\beta}\right)^{-1} \Gamma_{\beta}$, where $m_{\beta}$ is the measure and $\Gamma_{\beta}$ the characteristic function of $X_{\beta}$. Then $\left\|f_{\beta}\right\|=1$. Let $C_{\beta}=\mathrm{Cl}$ conv $\left\{f_{n} \mid n \geqq \beta\right\}$. Then $C_{\alpha}$ is a decreasing sequence of bounded closed convex sets whose intersection is empty, so by (I4.2), $L^{1} M$ is nonreflexive.

By infinite-dimensional $L^{p-s p a c e}$ we shall mean a space $L^{p} M$ where $1 \leqq p$ $<\infty$ and $M$ contains a sequence $X_{\alpha}$ as in (1.1). If $M=(X, m)$, where $X$ has cardinality $\aleph$ and $m$ is the counting measure on $X$, then $L^{p} M$ will be denoted by $l^{p} \aleph$ and will be called an infinite-dimensional $l^{p}$-space iff $\boldsymbol{\aleph}$ is infinite. We write $L M$ for $L^{1} M$ and $l \aleph$ for $l^{1} \aleph$.

From (I4.1) and (1.1) we obtain

(1.2) Suppose $E$ is a nonreflexive normed linear space 〈infinite-dimensional $L^{p}$-space $\rangle, B$ is a bounded convex body in $E$, and $Y$ is a weakly compact 〈compact $\rangle$ set such that $Y \subset \subset B$. Then there is a homeomorphism of $E$ onto $E \sim Y$ which is the identity on $E \sim$ Int $B$.

(1.3) Suppose $E$ is either a nonreflexive normed linear space or an infinitedimensional $L^{p}$-space and $U$ is the unit cell of $E$. Then there is a homeomorphism $h$ of $E \sim$ Int $U$ onto $U$ which is the identity on $F U$.

Proof. For each $x \in E \sim$ Int $U$, let $f_{1} x=\|x\|^{-2} x$. Then $f_{1}$ is a homeomorphism of $E \sim$ Int $U$ onto $U \sim\{\phi\}$ which is the identity on $F U$. Thus $h=f_{2} f_{1}$ is the desired homeomorphism, with $f_{2}$ as in (1.2) for $Y=\{\phi\}$.

Kakutani [13] showed that (in Hilbert space) $F U$ is a deformation-retract of $U$. From (1.3) we see that there is an almost- $h$-isotopy $\eta$ of $U$ into $U$ such that $\eta_{0}$ is the identity map on $U$ and $\eta_{1}$ is a retraction of $U$ onto $F U$.

(1.4) With E and U as in (1.3), there is a homeomorphism of period two without fixed points of $E$ onto itself which takes $U$ onto itself.

Proof. Let $h$ be as in (1.3). For $x \in E \sim U$, let $T x=-x$; for $x \in U$, let $T x=h\left(-h^{-1} x\right)$. Then $T$ is the desired homeomorphism.

(1.5) If $E$ is a nonreflexive normed linear space, then every hyperplane in $E$ is homeomorphic with the unit sphere $S$ of $E$.

Proof. Let $H$ be a hyperplane in $E$. We assume without loss of generality that $\phi \in H$, whence $H=[f ; 0] \equiv\{x \mid f(x)=0\}$ for some not-identically-zero continuous linear functional $f$ on $E$. From the characterization of reflexivity in terms of weak compactness it follows readily that $H$ must be nonreflexive, and hence by (1.3) that there is a homeomorphism $h$ of $H \sim \operatorname{Int} U$ onto $H \cap U$ which is the identity on $H \cap S$. Now let $y \in U \cap[f ;<0]$ and let $P$ be the 
projection from $y$ of $H \cap U$ onto $S \cap[f ; \geqq 0] . P$ is a homeomorphism which is the identity on $H \cap S$. Now for $x \in H \sim U$ let $T x=-P h x$; and $T x=P x$ for $x \in H \cap U$. Then $T$ is the desired homeomorphism.

(1.6) Each infinite-dimensional $L^{p}$-space is homeomorphic with its unit sphere.

Proof. Let $L^{p} M$ be the space under consideration. By using (1.1) and a known property of complete inner-product spaces we can find an infinite cardinal $\aleph$ and homeomorphisms $h_{i}$ such that $h_{1} L^{p} M=L^{2} M, h_{2} L^{2} M=l^{2} \aleph$, $h_{3} l^{2} \aleph=l^{1} \aleph$, and each $h_{i}$ preserves unit spheres. But $l^{1} \aleph$ is homeomorphic with each of its hyperplanes, so the proof is completed by (1.5).

(1.7) Suppose E is either a nonreflexive normed linear space or an infinitedimensional $l^{p}$-space, $U$ is the unit cell and $Q$ a closed half-space of $E$. Then there is a homeomorphism of $Q$ onto $U$ which takes $F Q$ onto $F U$.

Proof. We assume without loss of generality that $\phi$ is in the bounding hyperplane $F Q$ of $Q$. We then have, for some continuous linear functional $f$ on $E, F Q=[f ; 0]$ and $Q=[f ; \geqq 0]$. Obviously there is a homeomorphism $g_{1}$ of $Q$ onto $Q_{1}=[f ; \geqq 0] \cap[f ;<1]$ which is the identity on $F Q$. Pick $y \in[f ; 1]$ and let $h$ be the homeomorphism of $F Q$ onto $F U$ whose existence is guaranteed by (1.5) and (1.6). For each $x \in F Q$ let $g_{2}$ map the segment $\left.] y, x\right]$ linearly onto the segment $] \phi, h x]$. Then $g_{2}$ is a homeomorphism of $Q_{1}$ onto $U \sim\{\phi\}$ which takes $F Q$ onto $F U$. And by (1.2) there is a homeomorphism $g_{3}$ of $U \sim\{\phi\}$ onto $U$ which is the identity on $F U$. Thus $g_{3} g_{2} g_{1}$ is the desired homeomorphism of $Q$ onto $U$.

II2. An embedding property. This section establishes for certain sets an embedding property which is used in several proofs. Suppose $B$ is a metric space and $Y \subset B$. We shall say that $Y$ is conveniently situated in $B$ iff there is an open set $U \supset Y$ and a continuous map $D$ of $F U \times[0,1]$ into $\mathrm{Cl} U$ such that $U \subset N_{\epsilon} Y$ for some $\epsilon<\infty$ and

( $\alpha) D$ maps $F U \times] 0,1]$ topologically onto $\mathrm{Cl}(U) \sim Y$;

( $\beta$ ) for each $u \in F U, D(u, 1)=u$ and $D(u, 0) \in Y$;

( $\gamma)$ if $D\left(u_{\alpha}, t_{\alpha}\right) \rightarrow y \in Y$, then $t_{\alpha} \rightarrow 0$ and $D\left(u_{\alpha}, r_{\alpha}\right) \rightarrow y$ whenever $r_{\alpha} \rightarrow 0$.

If $B$ is a subset of a normed linear space we shall say that $Y$ is very conveniently situated in $B$ iff the neighborhood $U$ and function $D$ can be so chosen that $D(\{u\} \times[0,1])$ is a line-segment for each $u \in U$.

(II2.1) Suppose $E$ is a strictly convexifiable normed linear space and $K$ is a boundedly compact convex subset of $E$. Then $K$ is very conveniently situated in E.

Proof. We may as well suppose that $E$ is actually strictly convex. By (A2.1) and (A2.2) (see Appendix), $K$ has the nearness property in $E$ and $K$. is a retraction of $E$ onto $K$. For some $\epsilon>0$ let $U=N_{\epsilon} K$ and for each $u \in F U$ and $t \in[0,1]$ take $D(u, t)=t u+(1-t) K u$. Clearly $D$ is continuous, $(\beta)$ holds, and $D(\{u\} \times[0,1])$ is a line-segment for each $u \in F U$, so it remains only to verify $(\alpha)$ and $(\gamma)$. 
If $D\left(u_{\alpha}, t_{\alpha}\right)=t_{\alpha} u_{\alpha}+\left(1-t_{\alpha}\right) K u_{\alpha} \rightarrow y \in K$, then, by (A2.3), $K u_{\alpha}=K D\left(u_{\alpha}, t_{\alpha}\right)$ $\rightarrow y$. Thus if $t_{\alpha}$ does not $\rightarrow 0, u_{\alpha}$ must have a subsequence which converges to $y$, which is clearly impossible. Also, for $r_{\alpha} \rightarrow 0$ we have $\lim D\left(u_{\alpha}, r_{\alpha}\right)=\lim K u_{\alpha}=y$, so $(\gamma)$ has been established.

From (A2.4) (iii) it follows that $D$ maps $F U \times] 0,1]$ biuniquely onto $\mathrm{Cl} U \sim Y$. Now if $D\left(u_{\alpha}, t_{\alpha}\right) \rightarrow D(u, t)$, we have $K u_{\alpha} \rightarrow K u$ and hence $t_{\alpha} u_{\alpha}$ $-t_{\alpha} K u \rightarrow t u-t K u$. Now suppose $t>0$ and consider an arbitrary convergent subsequence of $t_{\alpha}, t_{n \alpha} \rightarrow s$. If $s=0$ we can conclude that $u=K_{u}$, which is impossible since $u \notin K$. And $s>0$ leads to $u_{n \alpha} \rightarrow t s^{-1} u+(s-t) s^{-1} K u$. If $s \neq t$ the limit point is interior to $U$, which is impossible since $F U$ is closed. Thus $t_{\alpha} \rightarrow t$; from this it follows readily that $u_{\alpha} \rightarrow u$. Hence $(\alpha)$ holds and the proof of (2.2) is complete.

We wish next to show that every finite polytope in a normed linear space must be very conveniently situated. For this we need (2.2) below.

(2.2) Suppose $E$ is a normed linear space and $B_{1}$ is a closed linear subspace of $E$ which has a closed complementary subspace $B_{2}$. Then if a set $Y$ is conveniently or very conveniently situated in $B_{1}$, it is similarly situated in $E$.

Proof. By hypothesis, each point $x \in E$ has a unique expression in the form $x_{1}+x_{2}$, with $x_{i} \in B_{i}$, and $x_{i} \mid x \in E$ is a continuous linear transformation of $E$ onto $B_{i}$. Now let $Y$ be either conveniently or very conveniently situated in $B_{1}$ and let $U$ and $D$ be the corresponding neighborhood and transformation. Let $C$ denote the unit cell of $B_{2}$. Then $V=U+\operatorname{Int} C$ is open in $E$ and $F V$ $=(F U+C) \cup(U+F C)$. For $t \in[0,1]$ and $p \in U$, define $G_{t}(p)$ as follows: if $p \in Y, G_{t}(p)=p$ for all $t$; if $p \notin Y$, and thus $p=D(u, s)$ for some $u \in F U$ and $s \in] 0,1], G_{t}(p)=D(u, t s)$. For each $v \in F V$ and $t \in[0,1]$, let $\widetilde{D}(v, t)=G_{t}\left(v_{1}\right)$ $+t v_{2}$. Then $\widetilde{D}$ is an extension of $D$. It is obvious that $\tilde{D}$ maps $F V \times[0,1]$ into $\mathrm{Cl} \mathrm{V}$, that (for each $v \in F V) \tilde{D}(v, 1)=v, \tilde{D}(v, 0) \in Y$, and $\tilde{D}(\{v\} \times[0,1])$ is a line segment whenever $D\left(\left\{v_{1}\right\} \times[0,1]\right)$ is. Continuity of $\tilde{D}$ follows from that of $v_{i} \mid v$ and $G_{t}\left(v_{1}\right) \mid\left(v_{1}, t\right)$, which is not difficult to establish. By a straightforward argument using the fact that $D$ satisfies $(\gamma)$ it can be seen that $\tilde{D}$ does also. Finally, it is tedious but not difficult to show that if $x \in \mathrm{Cl} V \sim Y$, then

for $x_{1} \in Y, \tilde{D}^{-1}(x)=\left(x_{1}+\left.\left\|x_{2}\right\|\right|^{-1} x_{2},\left\|x_{2}\right\|\right)$,

for $x_{1} \notin Y$ (and hence $x_{1}=D(u, s)$ for a unique $u \in F U$ and $\left.\left.s \in\right] 0,1\right]$ ),

$$
\begin{array}{ll}
\tilde{D}^{-1}(x)=\left(G_{\| x_{2}||^{-1}}\left(x_{1}\right)+\left\|x_{2}\right\|^{-1} x_{2},\left\|x_{2}\right\|\right), & \text { if }\left\|x_{2}\right\| \geqq s, \\
\tilde{D}^{-1}(x)=\left(u+s^{-1} x_{2}, s\right) & \text { if }\left\|x_{2}\right\| \leqq s .
\end{array}
$$

From these equations it follows that $\widetilde{D}$ satisfies $(\alpha)$ also, and the proof of (2.2) is complete.

The proof of (2.3) below was suggested by Dr. M. L. Curtis. It is simpler than the author's original proof.

(2.3) In a normed linear space, every finite polytope is very conveniently situated. 
Proof. Let $P$ be the polytope in question, $E$ the containing space, and $L$ the linear hull of $P$. Then $L$ is finite-dimensional and hence has a closed complementary subspace, so by (2.2) our problem is reduced to the case in which $E$ is a Euclidean $n$-space for some $n$. For a (simplicial) complex $S$ in $E^{n}$, $|S|$ will denote the union of all simplexes in $K$. Now for our polytope $P \subset E^{n}$ there are complexes $C$ and $K$ such that $C \subset K$ and $P=|C| \subset$ Int $|K|$. Let $C^{\prime}$ and $K^{\prime}$ be the barycentric derived complexes of $C$ and $K$, St $C^{\prime}$ the star of $C^{\prime}$ in $K^{\prime}$ and $U=\mid$ St $C^{\prime} \mid$. Then $U$ is open and from results of $[21$, p. 292] it follows that there is a continuous map $\rho$ of $F U$ onto $F\left|C^{\prime}\right|=F P$ such that the set $\mathrm{Cl}(U) \sim$ Int $P$ is simply covered by the family of segments $\{[u, \rho u] \mid u$ $\in F U\}$. Now for each $u \in U$ and $t \in[0,1]$ let $D(u, t)=t u+(1-t) \rho u$. It is easy to verify that $D$ satisfies $(\alpha),(\beta)$, and $(\gamma)$ and hence $P$ is very conveniently situated in $E$.

The property of conveniently situated sets which makes them useful for our purposes is expressed in the next result.

(2.4) Suppose $B$ is a metric space and $Y$ is conveniently situated in $B$ (via an open set $U$ and transformation $D)$. Let $\left.\left.L^{\prime}=\langle(B \sim Y) \times] 0,2\right]\right\rangle \cup\langle Y \times\{0,2\}\rangle$ and $L=\langle(B \sim Y) \times] 0,2]\rangle \cup\langle Y \times\{1,2\}\rangle$. Then there is a homeomorphism of $L^{\prime}$ onto $L$ which is the identity on $\langle(B \sim U) \times] 0,2[\rangle \cup\langle B \times\{2\}\rangle$ and takes $(y, 0)$ onto $(y, 1)$ for each $y \in Y$.

Proof. For $t \in[0,1]$ and $r \in[0,2]$ let

$$
\begin{gathered}
a(t, r)=\left\{\begin{array}{l}
2 t-1 \\
1 / 2 \\
t
\end{array}\right\} \text { and } b(t, r)=\left\{\begin{array}{l}
r \\
-2 t+3 r / 2+1 \\
1+r / 2
\end{array}\right\} \text { if } \\
\left\{\begin{array}{r}
r \leqq 4 t-2 \\
4 t-2 \leqq r \leqq 2 t \\
2 t \leqq r
\end{array}\right\} .
\end{gathered}
$$

Then the transformation $m:(t, r) \rightarrow(a(t, r), b(t, r))$ is a homeomorphism of $[0,1] \times[0,2]$ onto itself which is the identity on $\langle\{1\} \times[0,2]\rangle \cup\langle[0,1]$ $\times\{2\}\rangle$, and takes conv $\{(1,2),(1,0),(1 / 2,0)\}, \operatorname{conv}\{(1,2),(1 / 2,0),(0,0)\}$, and conv $\{(1,2),(0,0),(0,1)\}$ linearly onto conv $\{(1,2),(1,0),(0,0)\}$, conv $\{(1,2),(0,0),(0,1)\}$, and conv $\{(1,2),(0,1),(0,2)\}$ respectively.

Now let the transformation $G$ be the identity on $(B \sim U) \times[0,2], G(y, 0)$ $=(y, 1)$ for each $y \in Y$, and on $(\mathrm{Cl} U \sim Y) \times] 0,2]$ define $G$ by the equation $G(D(u, t), r)=(D(u, a(t, r)), b(t, r))$. We have defined $G$ at least once everywhere on $L^{\prime}$, and different definitions agree where both apply. It is clear that $g$ maps $L^{\prime}$ biuniquely onto $L$ and we wish to show that both $G$ and $G^{-1}$ are continuous. Except at points of $Y \times\{0\}$ and $Y \times\{1\}$ respectively, this follows readily from (i) above and the fact that $D$ is continuous.

To establish continuity of $G$ at $(y, 0)$ for $y \in Y$ we must show that if $D\left(u_{\alpha}, t_{\alpha}\right) \rightarrow y$ and $r_{\alpha} \rightarrow 0$, then $D\left(u_{\alpha}, a\left(t_{\alpha}, r_{\alpha}\right)\right) \rightarrow y$ and $b\left(t_{\alpha}, r_{\alpha}\right) \rightarrow 1$. Now from 
$(\gamma)$ above it follows that $t_{\alpha} \rightarrow 0$. But then from the definition of $m$ it follows that $m\left(t_{\alpha}, r_{\alpha}\right) \rightarrow(0,1)$, and by applying $(\gamma)$ again we see that, since $a\left(t_{\alpha}, r_{\alpha}\right)$ $\rightarrow 0, D\left(u_{\alpha}, a\left(t_{\alpha}, r_{\alpha}\right)\right) \rightarrow y$ and thus $G$ is continuous.

Suppose, on the other hand, that $D\left(u_{\alpha}, a\left(t_{\alpha}, r_{\alpha}\right)\right) \rightarrow y \in Y$ and $b\left(t_{\alpha}, r_{\alpha}\right) \rightarrow 1$. We see by $(\gamma)$ that $a\left(t_{\alpha}, r_{\alpha}\right) \rightarrow 0$ and thence from the definition of $a$ and $b$ that $t_{\alpha} \rightarrow 0$ and $r_{\alpha} \rightarrow 0$. Then (using $(\gamma)$ again) we see that $D\left(u_{\alpha}, t_{\alpha}\right) \rightarrow y$ and thus $G^{-1}$ is continuous.

II3. Further results.

(3.1) Suppose $Y$ is a subset of the normed linear space $E$ and at least one of the conditions below is satisfied. Then there is an h-isotopy $\eta$ of $E$ onto $E$ such that $\eta_{0}$ is the identity map on $E$ and $\eta_{1}$ projects $Y$ linearly into a hyperplane.

(i) $Y$ is convex and weakly compact, E cannot be covered by countably many weakly compact sets;

(ii) $Y$ is weakly compact, $E$ is nonreflexive and complete;

(iii) $Y$ is convex and compact, E cannot be covered by countably many compact sets;

(iv) $Y$ is compact, $E$ is infinite-dimensional and complete.

Proof. Note first that if (ii) holds then every weakly compact set is nowhere dense in $E$. (For otherwise the unit cell would be weakly compact and the space therefore reflexive.) And $E$, being complete, cannot be covered by countably many nowhere dense sets. Furthermore, by a theorem of Krein and Smulian [19, p. 581], $\mathrm{Cl}$ conv $Y$ is weakly compact, so $E$ and $\mathrm{Cl}$ conv $Y$ satisfy condition (i). Similarly, if (iv) holds then $E$ cannot be covered by countably many compact sets and by a theorem of Mazur [23], $\mathrm{Cl}$ conv $Y$ is compact. In this case, then, $E$ and $\mathrm{Cl}$ conv $Y$ satisfy condition (iii). In the rest of this proof we consider only cases (i) and (ii), since the others are similar but somewhat simpler.

Let $X=\mathrm{Cl}$ conv $Y$ (so that, in case (i), $X=Y$ ). Then $X-X$ is weakly compact and hence there is a point $p \in E \sim\{\phi\}$ such that Ray $[\phi, p[\cap(X-X)$ $=\{\phi\}$. (Otherwise $\cup_{n=1}^{\infty} n(X-X)=E$, which contradicts (i).) Since $X-X$ is convex and symmetric about $\phi$, this implies that no translate of the line $\{t p \mid-\infty<t<\infty\}$ can intersect $X-X$ more than once, and hence no translate intersects $X$ more than once. Thus (by translating the space if necessary) we can obtain a hyperplane $H=[f ; 0]$ complementary to this line and a multiple $q$ of $p$ such that $q \in[f ; 1], X \subset[f ;>2]$, and $X$ is mapped biuniquely into $H$ by the projection $P$ which takes $h+t q$ onto $h$ for each $h \in H$ and $t \in]-\infty, \infty[. P$ is linear and is continuous in both weak and strong topologies. Since $X$ is weakly compact, $P \mid X$ is actually a weak homeomorphism. We wish next to show that on $P X, P^{-1}$ is strongly continuous.

Consider an arbitrary sequence $h_{\alpha}$ in $P X$, with $h_{\alpha} \rightarrow h_{0}$ and $P^{-1} h_{\beta}=h_{\beta}$ $+t_{\beta} q$. We wish to show that $t_{\alpha} \rightarrow t_{0}$. If it does not, then by the boundedness and weak compactness of $X$ there must be a subsequence $n \alpha$, a number $t \neq t_{0}$, and a point $h+s q$ of $X$ such that $t_{n \alpha} \rightarrow t$ and $h_{n \alpha}+t_{n \alpha} q \rightarrow$ (weakly) $h+s q$. But 
$f\left(h_{\beta}+t_{\beta} q\right)=t_{\beta}$ and $f(h+s q)=s$, so from the weak convergence we have $t_{n_{\alpha}} \rightarrow s$ and thus $s=t$ and $h_{n \alpha} \rightarrow$ (weakly) $h$. Since also $h_{\alpha} \rightarrow h_{0}$, we then have $h=h_{0}$ and thus $h_{0}+t q$ and $h_{0}+t_{0} q$ are both in $X$. But $t \neq t_{0}$ and $P$ is singlevalued, so this is a contradiction showing that $P \mid X$ is a homeomorphism.

Now $P X$ is closed and convex, so by a theorem of Dugundji [7, p. 358] there is a retraction $\rho$ of $H$ onto $P X$. For each $h \in H$ let $f h$ be the (unique) number $s$ such that $\rho h+s q \in X$. Then $f$ maps $H$ continuously into ]2, $M$ [ for some $M<\infty$. Now for $s \in[0,1]$ and $h \in H$, let $\eta_{s}(h+t q)=h+t(1-s+s f h)^{-1} q$ for $t>0$ and $=h+t q$ for $t \leqq 0$. Then $\eta$ is the desired isotopy.

(3.2) Suppose $E$ is an infinite-dimensional normed linear space, $X$ a compact subset of $E$, and $M$ a compact metric space. Then every continuous map of $M$ into $E \sim X$ is homotopic to zero in $E \sim X$. If $E$ is complete, then every homeomorphism of $M$ into $E \sim X$ is almost-h-isotopic to zero in $E \sim X$.

Proof. If $E$ is noncomplete, then it is nonreflexive, and by (1.2) $E \sim X$ is homeomorphic with $E$, so the assertion of (3.2) is obvious. Now suppose $E$ is complete (and infinite-dimensional) and $h$ is a homeomorphism of $M$ into $E$. As in the proof of (3.1), there is a point $p \in E \sim\{\phi\}$ such that each translate of the line $\{t p \mid-\infty<t<\infty\}$ intersects $X \cup h M$ at most once. Let $[f ; c]$ be an arbitrary hyperplane in $E$ such that $X \subset[f ;<c]$ and $f p \neq 0$. Then for a sufficiently large $K>0$, the map $\eta(u, t)=h u+t K(f p) p \mid u \in M, t \in[0,1]$ is an $h$-isotopy of $M$ into $E \sim X$ such that $\eta_{1} M \subset[f ;>c+2]$. But then as in the proof of (3.1) it follows that $\eta_{1}$ is $h$-isotopic in $E \sim X$ to a homeomorphism $\eta_{2}$ of $M$ into $[f ; c+1]$, and it is clear that $\eta_{2}$ is almost-h-isotopic to zero in $[f ; \geqq c+1] \subset E \sim X$. Thus every homeomorphism of $M$ into $E \sim X$ is almost- $h$ isotopic to zero in $E \sim X$. The statement (for $E$ complete) about homotopies follows easily from this.

(3.3) Suppose $E$ is a normed linear space which is either nonreflexive or an infinite-dimensional $l^{p}$-space, $Q=[f ;>0]$ is an open half-space in $E$ with bounding hyperplane $H=[f ; 0], Y$ is a compact subset of $E$, and at least one of the following is true: (i) $Y$ is conveniently situated in some hyperplane in $E$; (ii) $Y$ is a finite polytope; (iii) $Y$ is convex, $E$ is complete and strictly convexifiable. Then if $Y \subset Q$, there is a set $Y^{\prime} \subset H$ and homeomorphism of $Q$ onto $Q \cup Y^{\prime}$ which takes $Y$ onto $Y^{\prime}$. If $Y \subset H$ and $X$ is a relatively open subset of $Y$, then there are homeomorphisms $h_{1}(Q \cup X)=Q$ and $h_{2}(Q \cup Y)=Q$ such that $h_{i}$ is the identity on $[f ; \geqq 2]$ and $h_{2}$ simply translates $Y$ into $[f ; 1]$.

Proof. If $Y \subset Q$, it is not difficult to describe (using (3.1)) in case (iii) a homeomorphism $h$ of $Q$ onto $Q$ such that $h Y$ is contained in a translate of $H$ and still satisfies either (i), (ii), or (iii). Thus the case $Y \subset Q$ is reduced to the case $Y \subset H$. Note also that if $X$ is a relatively open subset of $Y$, then $X \sim Y$ is compact and hence (3.3)'s assertion about $X$ follows from (1.2) and (3.3)'s assertion about $Y$. Furthermore, we see from (2.1) and (2.3) that cases (ii) and (iii) are included in case (i), so it remains only to show that if $Y$ is conveniently situated in $H$, then there is a homeomorphism of 
$Q \cup Y$ onto $Q$ which translates $Y$ into $Q$. This amounts to finding a homeomorphism $q$ of $\langle H \times] 0, \infty[\rangle \cup\langle Y \times\{0\}\rangle$ onto $H \times] 0, \infty$ [ such that $g(y, 0)$ $=(y, 1)$ for each $y \in Y$. And because of $(1.1)$ we can assume that $H$ is a nonreflexive normed linear space.

Since $Y$ is conveniently situated in $H$, there are a bounded open set $U \subset H$ and a mapping $D$ such that $(\alpha)-(\gamma)$ of $\$ 2$ are satisfied. Let $B$ be a bounded convex body in $H$ such that $\mathrm{Cl} U \subset B$. Then if $p \notin B$ or $t \notin[0,2]$, let $g(p, t)$ $=(p, t)$. It remains to define a homeomorphism of $\left.\left.Z_{1}=\langle B \times] 0,2\right]\right\rangle \cup\langle Y \times\{0\}\rangle$ onto $\left.\left.Z_{4}=B \times\right] 0,2\right]$ which is the identity on $\left.\left.S=\langle F B \times] 0,1\right]\right\rangle \cup\langle B \times\{2\}\rangle$. Let $\left.\left.Z_{2}=\langle(B \sim Y) \times] 0,2\right]\right\rangle \cup\langle Y \times\{0\}\rangle$ and $\left.\left.Z_{3}=\langle(B \sim Y) \times] 0,2\right]\right\rangle \cup\langle Y \times\{1\}\rangle$. From (2.4) it follows that there is a homeomorphism $h_{2}$ of $Z_{2}$ onto $Z_{3}$ which is the identity on $S$ and takes $(y, 0)$ into $(y, 1)$ for each $y \in Y$. Now if $\eta$ is as in the Isotopy Theorem (I1.1) and $\zeta$ as in (I1.2), then $\zeta^{*}$ is a homeomorphism of $B \times[0,1]$ onto $\langle(B \sim Y) \times] 0,1]\rangle \cup\langle B \times\{0\}\rangle$ which is the identity on $\langle F B \times[0,1]\rangle \cup\langle B \times\{0\}\rangle$. But then in an obvious way $\zeta^{*}$ can be used to define a homeomorphism $h_{1}$ of $Z_{1}$ onto $Z_{2}$ which is the identity on $S \cup(Y \times\{0\})$ and $\zeta^{*-1}$ can be used to define a homeomorphism $h_{3}$ of $Z_{3}$ onto $Z_{4}$ which is the identity on $S \cup(B \times\{1\})$. Then $g\left|B=h_{3} h_{2} h_{1}\right| B$ completes the desired definition of $g$ and the proof of (3.3).

We saw in (II1.1) that if $E$ is nonreflexive or an infinite-dimensional $L^{p}$-space, then an arbitrary compact set can be taken away from $E$ without changing its topological structure. In the theorem just proved, $Q$ is of course homeomorphic with $E$, so (3.3) shows, in a sense, that certain sets can be added to $E$ without changing its topological structure. Applied when $Y$ contains a single point, (3.3) will be fundamental in demonstrating that Hilbert space $\mathfrak{S}$ is homeomorphic with its unit cell. It will also be used in discussing periodic homeomorphisms of $\mathfrak{S}$.

If $X$ is a compact subset of $\mathfrak{S}$, then $\mathfrak{S} \sim X$ is homeomorphic with $\mathfrak{S}$ and hence is contractible. However, Blankinship [3] proved contractibility of $\mathfrak{S} \sim X$ assuming merely that $\mathrm{Cl} X$ is compact. By an easy application of (3.3) (iii) and Mazur's theorem [23] on compactness of a convex hull we have

(3.4) Suppose $E$ is a Banach space which is either nonreflexive or an infinitedimensional lp $l^{p}$ space, $X \subset E, p \in E \sim \mathrm{Cl}$ conv $X$, and $\mathrm{Cl}(X)$ is compact. Then the identity map on $E \sim X$ is almost-h-isotopic (in $E \sim X)$ to the map taking all points of $E \sim X$ into $p$.

\section{Chapter III. CONVEX BODIES AND PERIODIC HOMEOMORPHISMS}

\section{IN HILBERT SPACE}

III1. Convex bodies. In (II1.6) we saw that Hilbert space $\mathfrak{S}$ is homeomorphic with its unit sphere. We show here that it is also homeomorphic with its unit cell, and in fact that all closed convex bodies in $\mathfrak{S}$ are mutually homeomorphic. In (II1.7) it was proved that every closed half-space in $\mathfrak{E}$ is 
homeomorphic with the unit cell. And of course every open half-space in $\mathfrak{E}$ is homeomorphic with $\mathfrak{E}$ itself, so the next step will be to show that open halfspaces and closed half-spaces are topologically the same.

We work first with the space $L$ ] $0, \infty$ [ of Lebesgue integrable functions on ]0, $\infty$ [. The functions mentioned in (1.1) and (1.2) below are considered as points of this space, so $L X$ (for $X \subset$ ] $0, \infty$ [) means the subspace of $L] 0, \infty$ [ consisting of all functions in $L] 0, \infty$ [ which vanish almost everywhere on ] $0, \infty[\sim X$.

(1.1) There is an h-isotopy $\eta$ of $L] 0, \infty[$ almost-onto $L] 0, \infty\left[\right.$ such that $\eta_{1}$ is the identity map on $L] 0, \infty\left[\right.$ and $\left.\eta_{0} L\right] 0, \infty[=L] 1, \infty[$.

Proof. For each $t \in[0,1]$ and $f \in L] 0, \infty\left[\right.$, let $\eta_{t} f=f_{t}$, where $f_{t}$ is defined as follows: for $x \in] 0,1\left[, f_{t} x=t f(t x)\right.$; for $x \in\left[1, \infty\left[, f_{t} x=f(x+t-1)\right.\right.$. It is clear that each $\eta_{t}$ has the desired domain and range and that $\eta_{1}$ is the identity. We must show that $\eta^{*}$ is a homeomorphism.

Note first that for $t \in[0,1]$ and $\{f, \xi\} \subset L] 0, \infty[$,

$$
\begin{aligned}
\int_{0}^{1} t|f(t x)-\xi(t x)| d x+\int_{1}^{\infty} \mid f(x+t-1) & -\xi(x+t-1) \mid d x \\
& =\int_{0}^{t}|f-\xi|+\int_{t}^{\infty}|f-\xi|,
\end{aligned}
$$

whence

$$
\left\|f_{t}-\xi_{t}\right\|=\|f-\xi\|
$$

Also,

$\left\|f_{t}-f_{s}\right\|=\int_{0}^{1}|t f(t x)-s f(s x)| d x+\int_{1}^{\infty}|f(x+t-1)-f(x+s-1)| d x$.

Now if $f$ is a continuous function vanishing everywhere on ] $a, \infty[$ for some $a<\infty$, then from the uniform continuity of $f$ it follows readily that $\lim _{t \rightarrow s}\left\|f_{t}-f_{s}\right\|=0$. And for each $\left.\xi \in L\right] 0, \infty[$ and $\epsilon>0$ there is such a continuous $f$ for which $\|\xi-f\|<\epsilon$. (See, for example, [24, p. 229].) But then from (1) and the triangle inequality we see that $\left\|\xi_{t}-\xi_{s}\right\|<2 \epsilon+\left\|f_{t}-f_{s}\right\|$, and hence

$$
\lim _{t \rightarrow s}\left\|\xi_{t}-\xi_{s}\right\|=0 \text {. }
$$

We have $\left\|f_{t}-\xi_{s}\right\| \leqq\|f-\xi\|+\left\|\xi_{t}-\xi_{s}\right\|$ and $\|f-\xi\| \leqq\left\|f_{t}-\xi_{s}\right\|+\left\|\xi_{t}-\xi_{s}\right\|$, from which it follows by use of (2) that $\eta^{*}$ is a homeomorphism.

(1.2) $L] 0, \infty[\times[0,1]$ is homeomorphic with both $L] 0, \infty[\times] 0,1]$ and $L] 0, \infty[\times] 0,1[$.

Proof. Let $A=L] 0, \infty[\times[0,1], B=\langle L] 0, \infty[\times] 0,1]\rangle \cup\langle L] 1, \infty[$ $\times\{0\}\rangle$, and $C=\langle\mathrm{L}] 0,1[\times] 0,1]\rangle \cup\{(0, \phi)\}$. Then $\eta^{*}$ (as in (1.1)) is a homeomorphism of $A$ onto $B$. The map $m$ which takes $((t, f), g) \in C \times L] 1, \infty[$ 
onto $(t, f+g) \in B$ is a homeomorphism of $C$ onto $B$. By (II3.3), there is a homeomorphism of $C$ onto $L] 0,1[X] 0,1]$ which is the identity on $L] 0,1$ [ $\times\{1\}$, and this can be used to define a homeomorphism $h$ of $C \times L[1, \infty$ [ onto $L] 0,1[\times] 0,1] \times L] 1, \infty[$, which is homeomorphic with $L] 0, \infty[\times] 0$, 1] under the map $m$. Thus $m m^{-1} \eta^{*}$ is a homeomorphism of $\left.L\right] 0, \infty[\times[0,1]$ onto $L] 0, \infty[\times] 0,1]$ which is the identity on $M=L] 0, \infty[\times\{1\}$, and extending by reflection across $M$ yields a homeomorphism of $L] 0, \infty[\times[0,2]$ onto $L] 0, \infty[\times] 0,2[$, completing the proof of (1.2.)

Now since every hyperplane in $\mathfrak{S}$ is homeomorphic with $\mathfrak{S}$, as (by II1.1)) is $L$ ] $0, \infty$ [, by combining (II1.6), (II1.7), and (1.2) we obtain

(1.3) Hilbert space $\mathfrak{S}$ is homeomorphic with its unit sphere, its unit cell, $\mathfrak{S} \times] 0,1]$, and $\mathfrak{S} \times[0,1]$.

To show that all closed convex bodies in $\mathfrak{S}$ are mutually homeomorphic we employ the characteristic cone introduced by Steinitz and employed also by Stoker [29]. Let $K$ be a closed convex set in a normed linear space and $u$ a point of $K$. Then $K_{u} \equiv\{u\} \cup\{p \mid$ Ray $[u, p[\subset K\}$. It is readily verified that $K_{u}$ is a closed convex cone with vertex $u$, and that $K_{v}=K_{u}+(v-u)$ whenever $v \in K$. For any $u \in K, K_{u}$ is called a characteristic cone of $K$.

(1.4) Suppose $E$ is a normed linear space and $K$ is a closed convex body in $E$ whose characteristic cone is not a linear manifold. Then there is a homeomorphism of $K$ onto a closed half-space $Q$ which takes $F K$ onto $F Q$.

Proof. $S$ and $C$ will denote respectively the unit sphere and the unit cell of $E$. We assume without loss of generality that $\phi \in \operatorname{Int} K$. Then since $K_{\phi}$ is not a linear manifold, there is a point $y \in S$ such that Ray $[\phi, y] \subset$ Int $K$ but $-m y \in F K$ for some $m>0$. By the Mazur support theorem [15, (9.1)] there is a continuous linear functional $f$ with $\|f\|=1$ such that $f(-m y)$ $=\inf _{x \in K} f x$. Let $H=[f ; 0], C^{\prime}=\operatorname{conv}\langle(C \cap H) \cup\{y,-y\}\rangle, S^{\prime}=F C^{\prime}$, and let $\mu$ be the gauge function of $K$ relative to $\phi$. For each $s \in S^{\prime}$ and $t \geqq 0$ such that $t s \in K$ let $T(t s)=(1+\mu s) t(1+t)^{-1} s$. Then $T$ is a homeomorphism of $K$ onto Int $C^{\prime} \cup X$, where $X=S^{\prime} \sim\left(K_{\phi} \cap S^{\prime}\right) . X$ is relatively open in $S^{\prime}$ and (since $[f ; f(-m y)]$ bounds $K) X \supset[f ;<0] \cap S^{\prime}$. Now notice that $T$ maps the closed half-space $[f ; \geqq f(-m y)]$ onto $B \equiv \operatorname{Int} C^{\prime} \cup\left(S^{\prime} \cap[f ;<0]\right)$. Thus if we can map $T K$ topologically onto $B$ with Int $K$ mapping onto Int $C^{\prime}$, the proof of (1.4) will be complete.

Consider an arbitrary $x \in H \cap S=H \cap S^{\prime}$. There is a number $t_{x} \in[0,1[$ such that $t y+(1-t) x$ is in $X$ for $0 \leqq t<t_{x}$ but not for $t>t_{x}$. And always $\left[-y, x\left[\subset X\right.\right.$. Now let $G$ map $\left[y, t_{x} y+\left(1-t_{x}\right) x\right]$ linearly onto $[y, x],\left[t_{x} y\right.$ $\left.+\left(1-t_{x}\right) x, x\right]$ linearly onto $\left[x,-t_{x} y+\left(1-t_{x}\right) x\right]$, and $[x,-y]$ linearly onto $\left[-t_{x} y+\left(1-t_{x}\right) x,-y\right]$. Since $t_{x}$ is bounded from $1, G$ is a homeomorphism of $T K \cap S^{\prime}$ onto $B \cap S^{\prime}$, and thus the desired homeomorphism can be obtained by extending radially from $\phi$.

(1.5) Suppose $E$ is a normed linear space and $K$ is a closed convex body in $E$ with $\phi \in \operatorname{Int} K$. Suppose the characteristic cone $K_{\phi}$ of $K$ is a linear subspace $M$ 
which has a closed complementary subspace $M^{\prime}$. Let $C^{\prime}$ be the unit cell of $M^{\prime}$. Then there is a homeomorphism of $K$ onto $M \times X^{\prime}$ which takes $F K$ onto $M \times F C^{\prime}$.

Proof. We have $M \subset K$. Let $B=M^{\prime} \cap K$. Then $B$ is a closed convex body whose gauge function (relative to $\phi$ ) vanishes only at $\phi$ and we have $K=M$ $+B, F K=M+F B$ (with $F B$ being taken relative to $M^{\prime}$ ). It is easy to define a homeomorphism analogous to $T$ in the proof of (1.4) which maps $B$ and $F B$ onto $C^{\prime}$ and $F C^{\prime}$ respectively, and this completes the proof.

Applying (1.4) and (1.5) we obtain (1.6) below, which was proved for $E^{3}$ by Stoker [29].

(1.6) Suppose $K$ is a closed convex body in Euclidean $n$-space $E^{n}$. If the characteristic cone of $K$ is not a linear manifold, then $K$ is homeomorphic with $E^{n-1} \times\left[0,1\left[\right.\right.$ and $F K$ with $E^{n-1}$. If the characteristic cone is an m-dimensional linear manifold, then $K$ is homeomorphic with $E^{m} \times[0,1]^{n-m}$ and $F K$ with $E^{m} \times S^{n-m-1}$.

And applying (1.3)-(1.5) to $\mathfrak{S}$, we have

(1.7) Suppose $K$ is a closed convex body in $\mathfrak{W}$. Then $K$ is homeomorphic with $\mathfrak{W}$. If $K$ 's characteristic cone is not a linear manifold, or is a linear manifold of infinite deficiency in $\mathfrak{S}$, then $F K$ is also homeomorphic with $\mathfrak{S}$. If the characteristic cone is a linear manifold of deficiency $n$, then $F K$ is homeomorphic with $\$ \times S^{n-1}$.

It should be noticed that for $m<n, \mathfrak{S} \times S^{m}$ is not homeomorphic with $\mathfrak{S} \times S^{n}$. For every map of $S^{n}$ into $\mathfrak{S} \times S^{m}$ is homotopic to zero but the natural map of $S^{n}$ into $\mathfrak{S} \times S^{n}$ is not homotopic to zero.

Combining (1.6) and (1.7) with a result of Keller [14] quoted in §IV1, we have a complete description of all topological possibilities for a closed convex set $K \subset \mathfrak{E}$ provided $K$ either has an interior point, is compact, or is finite-dimensional.

III2. Periodic homeomorphisms. We shall first state some facts concerning periodic homeomorphisms in Euclidean $n$-space $E^{n}$, and then establish the results on Hilbert space stated in the introduction.

Let $T$ be a homeomorphism of period $k \geqq 2$ on $E^{n}$ and let $F$ be its fixedpoint set. If $n=3$, or $T$ is locally analytic and $n=4$, or $k$ is a power of a prime, then $F$ is nonempty and is "homologically similar" to $E^{m}$ for some $m \leqq n$. (For proofs and more precise statements see [26].) It is not known in general whether $F$ must be homeomorphic with some $E^{m}$ nor whether (for $n>4$ and $k$ not a prime power) $F$ must be nonempty.

For each $n$ there is an $\epsilon(n)>0$ such that $E^{n}$ does not admit a periodic homeomorphism having each orbit of diameter $<\epsilon(n)$. (This result, due to Smith [27], is a strengthened form of a theorem of Newman.)

On $E^{n}$, every pointwise periodic homeomorphism is periodic [25].

We shall say that a transformation is of pure period $n$ iff each non-fixed point is of period $n$ and there is at least one non-fixed point. A closed linear subset $L$ of $H$ will be called an intermediate subspace iff both $L$ and its or- 
thogonal complement are infinite-dimensional. If $L_{1}$ and $L_{2}$ are intermediate subspaces of $H$, then there is a linear isometry of $H$ onto itself which takes $L_{1}$ onto $L_{2}$.

(2.1) For each $n \geqq 2, H$ admits a linear isometry $T$ of pure period $n$ for which $\phi$ is the only fixed point.

Proof. Representing $H$ as $l^{2} \aleph_{0}$, let $T\left(x^{0}, x^{1}, x^{2}, \cdots\right)=\left(y^{0}, y^{1}, y^{2}, \cdots\right)$, where $y^{2 \beta}+i y^{2 \beta+1}=\exp (2 \pi i / n)\left(x^{2 \beta}+i x^{2 \beta+1}\right)$, or in other words,

$$
\begin{aligned}
y^{2 \beta} & =x^{2 \beta} \cos (2 \pi / n)-x^{2 \beta+1} \sin (2 \pi / n) \quad \text { and } \\
y^{2 \beta+1} & =x^{2 \beta} \sin (2 \pi / n)+x^{2 \beta+1} \cos (2 \pi / n) .
\end{aligned}
$$

(This transformation was suggested by the referee.) It is easy to verify that $T$ has the desired properties.

(2.2) For each $n \geqq 2, \mathfrak{S}$ admits a homeomorphism of pure period $n$ without fixed points.

Proof. Let $h$ be a homeomorphism of $\mathfrak{S}$ onto $\mathfrak{S} \sim\{\phi\}$ and let $S=h^{-1} T h$, where $T$ is as in (2.1). Then $S$ is the desired homeomorphism.

(2.3) Suppose $Y$ is compact and conveniently situated in a hyperplane in $\mathfrak{S}$ (or, in particular, that $Y$ is a finite polytope in $\mathfrak{S}$ ), $X$ is a relatively open subset of $Y$, and $n \geqq 2$. Then $\mathfrak{S}$ admits a homeomorphism of pure period $n$ whose fixedpoint set is $Y$, and also one of pure period $n$ whose fixed-point set is homeomorphic with $X$.

Proof. We may suppose that for some continuous linear functional $f$ on $\mathfrak{S}, Y \subset[f ; 1]$. Let $Q=[f ;>0]$ and $H=[f ; 0]$. By (II3.3) there is a set $Y^{\prime} \subset H$ and a homeomorphism of $Q$ onto $Q \cup Y^{\prime}$ which takes $Y$ onto $Y^{\prime}$. And if $X^{\prime}$ is the image of $X$ under this homeomorphism, then $Q \cup X^{\prime}$ is homeomorphic with $Q \sim(Y \sim X)$, which by (II2.1) is homeomorphic with $Q$. Since there is a homeomorphism of $\mathfrak{S}$ onto $Q$ which leaves each point of $Y$ fixed, our problem is reduced to that of describing on $Q \cup Y^{\prime}$ a homeomorphism of pure period $n$ with $Y^{\prime}$ as its set of fixed points.

By using $\eta^{*}$ of (1.1) and the homeomorphism in (II1.1) between $\left.L\right] 0, \infty$ [ and $L^{2}$ ],$\infty$ [ we can obtain a subset $Y^{\prime \prime}$ of an intermediate subspace $L_{1}$ of $H$ and a homeomorphism of $Q \cup Y^{\prime}$ onto $Q \cup Y^{\prime \prime}$ which takes $Y^{\prime}$ onto $Y^{\prime \prime}$. Let $L_{2}$ be the orthogonal complement in $\mathfrak{S}$ of $L_{1}$ and let $L_{3}$ be the intersection of $Q$ with the orthogonal complement in $\mathfrak{S}$ of $L_{1}+L_{2}$. ( $L_{3}$ is merely a ray.) Then $Q=L_{1}+L_{2}+\left(L_{3} \sim\{\phi\}\right)$. By the Isotopy Theorem (as used in the proof of (II3.3) to obtain a homeomorphism of $Z_{1}$ onto $\left.Z_{2}\right)\{\phi\} \cup\left[L_{2}+\left(L_{3} \sim\{\phi\}\right)\right]$ is homeomorphic with $\left.\{\phi\} \cup\left[L_{2} \sim\{\phi\}\right)+\left(L_{2} \sim\{\phi\}\right)\right]$, so there is a homeomorphism of $Q \cup Y^{\prime \prime}$ onto $\left[L_{1}+\left(L_{2} \sim\{\phi\}\right)+\left(L_{3} \sim\{\phi\}\right)\right] \cup Y^{\prime \prime}$ which is the identity on $Y^{\prime \prime}$. Now let $T$ be of pure period $n$ on $L_{2}$ with $\phi$ as its only fixed point (see 2.1) and for each $x=x_{1}+x_{2}+x_{3} \in Q \cup Y^{\prime \prime}$ (with $x_{i} \in L_{i}$ ) let $G x$ $=x_{1}+T x_{2}+x_{3}$. Then $G$ is of pure period $n$ and has $Y^{\prime \prime}$ as its set of fixed points, so the proof of $(2.3)$ is complete.

From (2.3) it follows that every open subset of a finite-dimensional 
Euclidean space can be topologically attained as the fixed-point set of a periodic homeomorphism in Hilbert space.

(2.4) Let $\epsilon$ be an arbitrary positive number, $n$ an arbitrary integer $\geqq 2$. Then $\mathfrak{S}$ admits a homeomorphism of pure period $n$ with fixed-point set $\{x \mid\|x\|$ $\geqq \epsilon\}$. (Here every orbit is of diameter $<2 \epsilon$, so Newman's theorem is not valid for $H$.)

Proof. From (II1.7) it follows that there is a homeomorphism of $\mathfrak{S}$ onto itself which takes $\{x \mid\|x\|=\epsilon\}$ onto a hyperplane $[f ; 0]$ and $\{x \mid\|x\| \geqq \epsilon\}$ onto $[f ; \leqq 0]$. Let $L$ be an intermediate subspace of $[f ; 0]$ and $p \in[f ; 1]$. By an argument analogous to that of (2.3) there is a homeomorphism of $\mathfrak{S}$ onto

$$
A=\langle L+\{t p \mid t \leqq 0\}\rangle \cup\langle[f ;>0] \sim(L+\{t p \mid t>0\})\rangle
$$

which takes $[f ; \leqq 0]$ onto the left-hand set, and then $A$ admits a homeomorphism of pure period $n$ with this as its fixed-point set, so the proof is complete.

A slight elaboration of the same argument proves

(2.5) Let $f$ be a not-identically-zero continuous linear functional on $\mathfrak{S}, G$ an open subset of $]-\infty, \infty[, \Gamma$ the set of all components of $G$, and $p$ a function on $\Gamma$ to the set of integers $\geqq 2$. Then $\mathfrak{S}$ admits a homeomorphism with fixed-point set $f^{-1}(]-\infty, \infty[\sim G)$ which for each $C \in \Gamma$ is of pure period $p C$ on $f^{-1} C$.

\section{Chapter IV. Additional Results and Problems}

1. Compact convex sets. Some of our theorems on closed convex bodies are analogous to the following results of Keller [14] on compact convex sets: Let $\Omega$ be the class of compact convex infinite-dimensional subsets of Hilbert space. Then all sets in $\Omega$ are topologically equivalent. The Hilbert parallelotope $P$ (and hence every set in $\Re$ ) is homogeneous; that is, for each pair of points $x$ and $y$ of $P$ there is a homeomorphism $h P=P$ such that $h x=y$. We indicate here a proof of the following extension of Keller's first result.

(1.1) Let $\Omega^{\prime}$ be the class of all sets $K$ having the following property: For some separable Banach space $E$ having a basis, $K$ is a compact convex subset of $E$ which is not contained in any hyperplane. Then all sets in $\Omega^{\prime}$ are topologically equivalent.

From (1.1) and Keller's second result it follows that every set in $\Omega^{\prime}$ is homogeneous.

The basic idea and most of the details of Keller's proof apply in proving (1.1). However, certain modifications are necessary, and we proceed to develop the necessary preliminary results.

If $X$ is a convex set in a normed linear space and $x \in X$, then $x$ is called a non-support point of $X$ iff there is no hyperplane which bounds $X$ and contains $x$. Following Keller, $X$ will be called elliptically convex provided the segment ] $x, y$ [ consists entirely of non-support points of $X$ whenever $x$ and $y$ are distinct points of $X$. 
(1.2) Suppose $X$ is a bounded convex subset of the strictly convexifiable normed linear space $E$, and that $X$ contains at least one non-support point. Then $X$ has an elliptically convex homeomorph in $E$.

Proof. We may assume that $\phi$ is a non-support point of $X$ and that the norm \|\| in $E$ is strictly convex. Since $X$ is bounded, we have $X \subset N_{d} \phi$ for some positive $d$. Now let $g$ be a real-valued, continuous, concave, and strictly monotone decreasing function on the interval $[0, d]$, with $g 0>0$ and $t g t \mid t$ strictly monotone increasing. (With $0<b<(2 d)^{-1}, 1-b t \mid t \in[0, d]$ is such a function.) Let $G x=(g\|x\|) x$ for each $x \in N_{a} \phi$.

Since $\operatorname{tgt} \mid t$ is strictly increasing, $G$ defines a biunique transformation of $N_{a} \phi$ onto $N_{d g a} \phi$. Clearly $G$ is continuous. We show next that $G^{-1}$ is continuous. Suppose $y_{\alpha} \rightarrow y_{0}$, with $y_{\beta}=G x_{\beta}$. We wish to show that $x_{\alpha} \rightarrow x_{0}$. And since each point maps onto a multiple of itself, it suffices to show that $\left\|x_{\alpha}\right\| \rightarrow\left\|x_{0}\right\|$. But this follows at once from the fact that $t g t \mid t$, being strictly monotone, defines a homeomorphism of $[0, d[$ onto $[0, d g d[$. We still must show that $Y=G X$ is elliptically convex. Consider any two points $u$ and $v$ of $X$ and an. arbitrary point $z \in] G u, G v[$. Let $\alpha=g\|u\|>0$ and $\beta=g\|v\|>0$. Then there are a point $y \in] u, v[$ and a number $t \in] 0,1$ [ such that $y=t u+(1-t) v$ and $z=m y$, with $m=\alpha \beta /[\beta t+\alpha(1-t)]$. We have

$$
g\|y\|=g(\|t u+(1-t) v\|)>g(t\|u\|+(1-t)\|v\|) \geqq t \alpha+(1-t) \beta \geqq m,
$$

where the first inequality is justified by the strict convexity of \|\| and strict monotoneity of $g$, the second by the concavity of $g$.

We now have $m y \in] \phi, G y[\subset G X$. Since $\phi$ is clearly a non-support point of $Y=G X$, so is $m y$, since any hyperplane which bounded $Y$ and contained $m y$ would also contain $\phi$, which is impossible. Hence $Y$ is elliptically convex and the proof is complete.

(1.3) Suppose $S$ is a separable Banach space which has a basis, $K$ is a convex subset of $S$, and $\phi$ is a non-support point of $K$. Then $S$ has a basis $\left\{y_{1}, y_{2}, \cdots\right\}$ such that for each $i,\left[\phi, y_{i}\right] \subset K$.

Proof. Let $\left\{x_{1}, x_{2}, \cdots\right\}$ be an arbitrary basis for $S$. By a theorem of Krein, Milman, and Rutman [18], there is a sequence $\delta_{\alpha}$ of positive numbers such that if $\left\|y_{\beta}-x_{\beta}\right\|<\delta_{\beta}$ then $\left\{y_{1}, y_{2}, \cdots\right\}$ is a basis for $S$. Let $C_{\beta}$ be the star from $\phi$ over $N_{\delta_{\beta}} x_{\beta}$. If $K$ does not intersect $C_{\beta}$, it follows by a theorem of Tukey $[15,(9.1)]$ that there is a hyperplane $H$ which separates $K$ and $C_{\beta}$. But then $H \ni \phi$, which contradicts the fact that $\phi$ is a non-support point of $K$. Thus if $y_{\beta} \in K \cap C_{\beta},\left\{y_{1}, y_{2}, \cdots\right\}$ is the desired basis.

Proof. of (1.1). Consider first a single set $K \subset \Omega^{\prime}$, and let $E$ be the space described in (1.1). From theorem (12.1) in [15] (which is an extension of Keller's Theorem 3) it follows that $K$ has a non-support point. A theorem of Clarkson [6] (and also (A1.10)) asserts that every separable Banach space is strictly convexifiable, so by $(2.2) K$ has an elliptically convex homeomorph $K_{1}$ in $E$ such that $\phi$ is a non-support point of $K_{1}$. Then by (1.3) there 
is basis $\left\{y_{1}, y_{2}, \cdots\right\}$ for $E$ such that $[\phi, y] \subset K$.

Now given another set in $\AA^{\prime}, K^{\prime} \subset E^{\prime}$, we define $K_{1}^{\prime}$ and $\left\{y_{1}^{\prime}, y_{2}^{\prime}, \ldots\right\}$ in an analogous fashion, and then Keller's proof (pp. 754-756) applies almost without change to define a homeomorphism of $K_{1}$ onto $K_{1}^{\prime}$.

IV2. Some comments and questions. This section is devoted to a discussion of what seem to the author to be some of the most important remaining questions concerning the topology of linear spaces.

Perhaps the most basic question is the following: Are all infinite-dimensional separable Banach spaces mutually homeomorphic? Very little is known concerning this question, and it even appears to be unknown whether the space of continuous real functions on the unit square is homeomorphic with that on the unit interval. Mazur's result [22] on homeomorphy of $L^{p}$-spaces has been extended by Kaczmarz [12] and M. H. Stone [30] to wider classes of spaces. Borsuk [4] has proved that if $W_{n}$ is the Banach space of all real continuous functions on the $n$-cell, then $W_{n} \times W_{n}$ is isomorphic (and hence homeomorphic) with $W_{n}$. The author [17] has proved that a Banach space cannot be homeomorphic with a non-complete normed linear space.

The more detailed results of this paper have been proved only for nonreflexive and infinite-dimensional $L^{p}$-spaces. In order to extend their scope, it would be of interest to know that every infinite-dimensional Banach space admits a homeomorphism onto a space in this favored class, and if possible a homeomorphism which takes the unit sphere of the first onto that of the second.

Although much less fundamental, the following problem on the homeomorphy of linear spaces may be of interest. Let $E$ be a smooth reflexive Banach space. Then for each $x \in E$ there is an unique $f_{x} \in E^{*}$ such that $\left\|f_{x}\right\|$ $=\|x\|$ and $f_{x} x=\left\|f_{x}\right\|\|x\|$. From the weak compactness of $E$ and $E^{*}$ it follows that the transformation $T: x \rightarrow f_{x}$ takes $E$ onto $E^{*}$ and is continuous as a map of $E$ onto $E_{w}^{*}$ ( $E^{*}$ in its weak topology.) Now if $E$ is also strictly convex then $E^{*}$ is smooth and $T$ defines a biunique transformation of $E$ onto $E^{*}$, $T$ being continuous on $E$ to $E_{w}^{*}$ and $T^{-1}$ being continuous on $E^{*}$ to $E_{w}$. Problem: Characterize intrinsically those spaces $E$ for which $T$ is a homeomorphism of $E$ onto $E^{*}$ (or of $E_{w}$ onto $E_{w}^{*}$ ).

In view of (II3.3) and (III2.3) it would be of interest to extend as far as possible the class of subsets of $\mathfrak{S}$ which are known to be conveniently situated, or to be homeomorphic with a conveniently situated subset of $\mathfrak{S}$.

It is desirable to remove the assumption of a basis in (IV1.1). One way to do this would be to prove that every separable Banach space $E$ has a basis over the space $\boldsymbol{I N}_{0}$, in the following sense; there is a sequence of elements $x_{\alpha}$ of $E$ such that if $t_{\alpha}$ is a sequence of real numbers having $\Sigma\left|t_{\alpha}\right|<\infty$ and $\Sigma t_{\alpha} x_{\alpha}=\phi$, then $t_{\beta}=\phi$. Keller's result and those of \$III1 describe all topological possibilities for a closed convex subset of $H$ which is either compact, 
finite-dimensional, or has an interior point. What can be said without this last restriction? Are there still only countably many possibilities? Is every infinite-dimensional closed convex set homogeneous? What stronger homogeneity properties are possessed by the Hilbert parallelotope?

We have seen (III1.3) that if $X$ is an $n$-cell, then $X \times \mathfrak{S}$ is homeomorphic with $\mathfrak{S}$. What other Peano continua have this property? A triod? An arbitrary dendrite?

Suppose $K$ is a compact subset of $\mathfrak{S}$ and $h$ is a homeomorphism of $K$ into $\mathfrak{S}$. Can $h$ be extended to a homeomorphism of $\mathfrak{S}$ onto $\mathfrak{S}$ ?

To conclude this section, we prove below a theorem on isotopies in $\mathfrak{S}$, the proof due jointly to Prof. James Dugundji and the author. This theorem stimulates the following question: Suppose $h_{0}$ and $h_{1}$ are homeomorphisms of $\mathfrak{S}$ onto $\mathfrak{S}$. Is there an isotopy $\eta$ of $\mathfrak{S}$ onto $\mathfrak{S}$ such that $\eta_{0}=h_{0}$ and $\eta_{1}=h_{1}$ ?

(2.1) If $M$ is a metric space, then the homeomorphisms of $M$ into $\mathfrak{S}$ are all mutually $h$-isotopic.

Proof. Let $L_{1}, L_{2}$, and $L_{3}$ be linear subspaces of $\mathfrak{S}$ such that $L_{1}$ and $L_{2}$ are intermediate subspaces, $L_{3}$ is one-dimensional, and $L_{i} \cap L_{j}=\{\phi\}$ for $i \neq j$. For $x \in \mathfrak{S}$, let $x=x_{1}+x_{2}+x_{3}$ be the unique representation of $x$ with $x_{i} \in L_{i}$. Now let $y \in L_{3} \sim\{\phi\}, Z_{0}=L_{1}$, and $Z_{1}=L_{2}+y$. Let $\mu$ be the identity map on $\mathfrak{S}$. By (III1.1) there are $h$-isotopies $\zeta^{i}$ of $\mathfrak{S}$ into $\mathfrak{S}$ such that $\zeta_{1}^{i}=\mu$ and $\zeta_{0}^{i} H=Z_{i}$. Then with $\psi_{t}^{i}=\zeta_{i}^{i} h_{i}$, it is easy to verify that $\psi^{i}$ is an $h$-isotopy of $M$ into $\mathfrak{S}$ such that $\psi_{1}^{i}=h_{i}$ and $\psi_{0}^{i} M \subset Z_{i}$. With this in view, we need consider henceforth only the case in which $h_{i} M \subset Z_{i}$.

Now in this case, let $\eta(p, t)=(1-t) h_{0} p+t h_{1} p$ for each $t \in[0,1]$ and $p \in M$. It is clear that $\eta$ is continuous. And since $\eta(p, t)_{1}=(1-t) h_{0} p, \eta(p, t)_{2}$ $=t\left[h_{1} p-y\right]$, and $\eta(p, t)_{3}=t y$, it is easily verified that $\eta$ is biunique and $\eta^{-1}$ is continuous. Thus $\eta$ is a homeomorphism, whence it follows easily that $\eta^{*}$ is a homeomorphism, completing the proof.

\section{APPENDIX. STRICT CONVEXITy AND THE NEAREST-POINT MAPPING}

A1. Remarks on smoothness and strict convexity. A convex body will be called strictly convex iff its boundary contains no line segments and smooth iff it has a unique supporting hyperplane through each boundary point. In a sense to be made precise later in this section, there is a strong duality between these notions. Thus we adopt the notational convention that if $S$ denotes one of the adjectives (strictly convex, smooth) $S^{*}$ will denote the other. A normed linear space will be called $S$ iff its unit sphere is $S$, and $S$-able (strictly convexifiable, smoothable) iff it is isomorphic (in the sense of Banach [1, p. 180]) with an $S$ space. In connection with several results of this paper, and also with certain other questions of functional analysis, it is of interest to extend as far as possible the class of spaces known to be strictly convexifiable. (It appears unlikely that every space is in the class, but this question remains unanswered.) In the present section, we extend the class 
beyond what was previously known and establish some characterizations of $S$-able spaces.

(A1.1) If $E^{*}$ is $S^{*}$, then $E$ is $S$.

Proof. Let $C$ and $S\left\langle C^{*}\right.$ and $\left.S^{*}\right\rangle$ be respectively the unit cell and unit sphere of $E\left\langle E^{*}\right\rangle$. If $C$ is not smooth, then for some $x \in S$ there are distinct functionals $f, g \in S^{*}$ for which $f x=g x=1$. But then $S^{*}$ contains the segment $[f, g]$ and is not strictly convex. If $C$ is not strictly convex, it contains a segment $\left[x_{1}, x_{2}\right]$. Let $f$ in $S^{*}$ be such that $f[(x+y) / 2]=1$. Then $f \equiv 1$ on $\left[x_{1}, y_{2}\right]$ and $\left\{g \mid g \in E^{*}\right.$ and $\left.g x_{i}=1\right\}$ are different hyperplanes supporting $C^{*}$ at $f$, so $C^{*}$ is not smooth. This completes the proof.

(A1.2) If $E^{*}$ is $S^{*}$-able by means of a $w^{*}$-compact unit cell, then $E$ is $S$-able.

Proof. Let $C=\left\{x \mid f x \leqq 1\right.$ for all $\left.f \in C^{*}\right\}$, where $C^{*}$ is the $w^{*}$-compact set mentioned in the hypothesis. Let $E_{1}\left\langle E_{2}\right\rangle$ be the space $E\left\langle E^{*}\right\rangle$ as renormed by the gauge function (relative to the neutral element) of $C\left\langle C^{*}\right\rangle$. We know that $E_{2}$ is $S$ and is isomorphic with $E^{*}$. It is easy to verify that $E_{1}$ is isomorphic with $E$, and from the $w^{*}$-compactness of $C^{*}$ it follows that $E_{1}^{*}=E_{2}$. The proof is completed by (A1.1).

From (A1.1) and (A1.2) we obtain

(A1.3) Suppose $E$ is reflexive. Then $E$ is $S\langle S$-able $\rangle$ if and only if $E^{*}$ is $S^{*}\left\langle S^{*}\right.$-able $\rangle$.

(A1.4) The following statements are equivalent: (i) $E$ is strictly convexifiable; (ii) E contains a convex body which is strictly convex near one of its boundary points; (iii) some convex open set in $E$ admits a strictly convex continuous functional; (iv) $E$ admits a norm $N \leqq\|\|$ such that $(E, N)$ is strictly convex; (v) there is a biunique continuous linear transformation $T$ on $E$ such that $T E$ is strictly convexifiable; (vi) among the bounded closed convex bodies in $E$ (metrized by the Hausdorff distance) the strictly convex ones are dense.

Proof. (ii) implies (iii). Let $C$ be the body described in (ii), $\phi$ the boundary point in question, $H$ a hyperplane supporting $C$ at $\phi, p \in \operatorname{Int} C$, and $P$ the projection of $E$ onto $H$ which maps $p$ onto $\phi$. For a sufficiently small open convex $U \ni \phi, U \cap F C$ contains no line segments and $P$ maps $U \cap F C$ homeomorphically onto a convex open $V \ni \phi$. For each $h \in V$ let $\eta h$ be such that $h+(\eta h) p \in U \cap F C$. Then $\eta$ is a strictly convex continuous functional defined on $V$, and $\eta h+t^{2} \mid h+t p$ is such a functional defined on $V$ $+]-\infty, \infty[p$, completing the proof.

(iii) implies (iv). Let $\eta$ be a strictly convex continuous functional defined on a convex open set $U \ni \phi$. Set $W=U \cap(-U)$ and for each $x \in W$ let $\psi x=\sup (\eta(x), \eta(-x))$. Then $\psi$ is strictly convex and attains its minimum $m$ only at $\phi$. For $\epsilon>0$, let $C_{\epsilon}=\{x \mid \psi x \leqq m+\epsilon\}$. Each $C_{\epsilon}$ is convex and has $\phi$ as an interior point. For $\epsilon$ sufficiently small, $\mathrm{Cl} \mathrm{C}_{\epsilon} \subset W$, and in this case $F C_{\epsilon}=\{x \mid \psi x=m+\epsilon\}$, so $C_{\epsilon}$ is strictly convex. Thus for $t$ sufficiently large, the gauge function (from $\phi$ ) of $t C_{\epsilon}$ is the desired $N$.

(iv) implies (i). With $N$ as in (iv), $(E, N+\|\|)$ is a strictly convex iso- 
morph of $(E,\|\|)$.

Thus we have shown that (i), (ii), (iii), and (iv) are mutually equivalent. Clearly (iv) and (v) are equivalent and (vi) implies (iii); to complete the proof we show that (i) implies (vi). Let $(E, M)$ be a strictly convex isomorph of $(E,\|\|)$. Consider an arbitrary positive $\epsilon$ and bounded closed convex body $C$ in $E$ such that $\phi \in \operatorname{Int} C$. Let $\mu$ be the gauge function of $C$ relative to $\phi$ and for each $\delta>0$ let $C_{\delta}=\{x \mid \mu x+\delta M x \leqq 1\}$. Then each $C_{\delta}$ is a closed strictly convex body contained in $C$. It can be verified that for $\delta$ sufficiently small, $C \subset N_{\epsilon} C_{\delta}$, completing the proof of (A1.4).

Similar techniques serve to establish (A1.5)-(A1.6) below.

(A1.5) For the statements below-(i) and (ii) are equivalent and are both implied by (iii) and by (iv). If $E$ is reflexive, all four are equivalent. (i) $E$ is smoothable; (ii) $E$ contains a bounded smooth convex body; (iii) $E$ contains a bounded convex body which is smooth near one of its extreme points; (iv) among the bounded closed convex bodies in $E$, the smooth ones are dense.

(A1.6) For the statements below-(i) and (ii) are equivalent and are both implied by (iii). If $E$ is reflexive, all three are equivalent. (i) $E$ has an isomorph which is both $S$ and $S^{*}$; (ii) E contains a bounded closed convex body which is both $S$ and $S^{*}$; (iii) among the bounded closed convex bodies in $E$, those which are both $S$ and $S^{*}$ are dense.

We next establish

(A1.7) Suppose $E$ is a reflexive Banach space. Then if $E$ is $S$-able, so is every continuous linear image of $E$.

Proof. We may as well suppose $E$ to be $S$. Let $C$ be the unit cell of $E$ and let $T E=E_{1}$ be a continuous linear transformation. Then $T$ is also weakweak continuous, so $T C$ must be weakly compact and hence closed. Since $T$ is interior [1, p. 40], $T C$ is a bounded closed convex set in $E_{1}$, containing $\phi_{1}$ in its interior and such that $-T C=T C$. Thus the gauge function of $T C$ is an admissible norm for $E_{1}$. Since ( $T C$ being closed) the fact that $C$ is $S$ implies readily that $T C$ is $S$, the proof is complete.

(A1.8) For each cardinal $\boldsymbol{N}, \boldsymbol{N}$ is strictly convexifiable. For each $\epsilon>0$, $\mathbf{N}_{0}$ admits a norm $N$ in which it is both smooth and strictly convex and such that $(\dagger) 0 \leqq\|f\|_{1}-N f<\epsilon\|f\|_{1}$ for each $f \in l \aleph_{0}$.

Proof. For each $f \in l \mathbb{N}$, let $M f=\|f\|_{1}+\|f\|_{2}$. Then \|\|$_{1} \leqq M \leqq 2\|\|_{1}$ and ( $l \boldsymbol{N}, M)$ is strictly convex. (Here $\|f\|_{p}$ is the norm of $f$ as a member of $l^{p} \aleph$.)

To handle the case of $l \boldsymbol{N}_{0}$, let $r$ be such that $0<r<\min (1, \epsilon) / 2$ and $\epsilon_{\beta}>0$ such that $1-r^{\beta \epsilon_{\beta}}<r^{\beta}$. Take $\xi f=\sum_{1}^{\infty}|f i|^{1+\epsilon_{i}}, C=\{f \mid \xi f \leqq 1\}$, and let $N$ be the gauge-function (from $\phi$ ) of $C$. If $\xi f \leqq 1$, then each $|f \beta| \leqq 1$ and hence

$$
\begin{aligned}
0 \leqq\|f\|_{1}-\xi f & =\sum_{1}^{\infty}|f i|\left(1-|f i| \epsilon_{i}\right) \leqq \sum_{|f i| \leqq r^{i}} r^{i}+\sum_{|f i|>r^{i}}\left(1-r^{i \epsilon_{i} i}\right) \\
& \leqq \sum_{1}^{\infty} r^{i}=r /(1-r)<2 r<\epsilon .
\end{aligned}
$$


Now when $N f=1$ we have $\xi f=1$ and $\|f\|_{1} \geqq 1$, so $(\dagger)$ holds. Due to homogeneity of the inequality $(\dagger)$, this establishes $(\dagger)$ for all $f$.

Now each function $\left|f_{i}\right|^{1+\varepsilon_{i}} \mid f$ is convex. Hence so is $\xi$ and thus $C$ is convex and closed. Then $N$ is an admissible norm for $l \boldsymbol{\aleph}_{0}$ and $C$ is the unit cell for $N$. Now if $g$ and $h$ are distinct points of $F C$, then for some $j, g j \neq h j$. Since $t^{1+\epsilon} j \mid t$ is strictly convex, $] g, h[\subset$ Int $C$ and $C$ is strictly convex.

Now let $g$ be a boundary point of $C, H$ a hyperplane supporting $C$ at $g$, and $\eta$ the unique linear functional which is equal to 1 everywhere on $H$. Then $\eta \leqq \xi$ and $\eta g=\xi g=1$. Now for each $j$ let $\delta_{j}$ be the characteristic function (on the set of all positive integers) of $\{j\}, u_{j}=\xi\left(g+t \delta_{j}\right) \mid t$ real, and $v_{j}$ $=\eta\left(g+t \delta_{j}\right) \mid t$ real. Then $v_{j}$ is linear, $v_{j} \leqq u_{j}$ and $v_{j} 0=u_{j} 0=1$. Since $u_{j}$ is differentiable, $v_{j}$ is completely determined by these conditions. Thus $\eta\left(g+t \delta_{j}\right)$ is uniquely determined for all $t$ and $j$, so $\eta$ (and hence $H$ ) is uniquely determined and $C$ is smooth.

In relation to (A1.7) and (A1.8), it is of interest to note that every Banach space is a continuous linear image of some $\boldsymbol{l} \mathbf{N}$, and every separable Banach space is a continuous linear image of $\boldsymbol{\aleph}_{0}[2$, p. 111].

(A1.9) Suppose $X$ is a topological space which admits a measure $m$ defined at least for all open sets and such that $m X=1$ and $m G>0$ for each nonempty open $G \subset X$. Then the space $C X$ of all bounded real-valued functions on $X$ is strictly convexifiable.

Proof. The usual norm \|\| of $C X$ is defined by $\|f\|=\sup _{x \in X} f x$. Let $N f$ $=\left[\int_{X} f^{2} d m\right]^{1 / 2}$. Then $N \leqq \|$ and $(C X, N)$ is strictly convex, so $(C X,\|\|+N)$ is a strictly convex isomorph of $(C X,\|\|)$.

(A1.10) If $E$ is a separable Banach space, both $E$ and $E^{*}$ are strictly convexifiable.

Proof. As observed in [2, p. 107], both $E$ and $E^{*}$ are equivalent to linear subsets of the space $(m)$, and strict convexifiability of $(m)$ follows from (A1.9).

That separable spaces are all strictly convexifiable was proved differently by Clarkson [6, p. 413].

From (A1.10) and (A1.3) we obtain

(A1.11) Every separable reflexive Banach space is both $S$-able and $S^{*}$-able.

(A1.12) If $X$ is a compact Hausdorff space which is an absolute retract relative to such spaces, then $C X$ is strictly convexifiable.

Proof. $X$ can be topologically embedded in a parallelotope $P$. Let $\rho$ be a retraction of $P$ onto $X$, and for each $f \in C X$ let $T f$ be the function $f \rho y \mid y \in P$. Then $T$ is an isometry of $C X$ into $C P$. By (A1.9), $C P$ is strictly convexifiable, and thus so is $C X$.

(A1.13) Suppose $E$ is a normed linear space and $X$ is the unit cell of $E^{*}$ in its *-weak topology. If $X$ is an absolute retract relative to compact Hausdorff spaces, then $E$ is strictly convexifiable.

Proof. For each $x \in F$ let $T x$ be the function $f x \mid f \in X$. Then $T$ is a linear isometry of $E$ into $C X$, so the proof is completed by (A1.12). 
To see that (A1.13) is not universally applicable to establish strict convexifiability, let $X$ be the unit cell of $l^{2} \mathbf{\aleph}_{1}^{*}\left(=l^{2} \mathbf{\aleph}_{1}\right)$ in the weak topology. As naturally embedded in the parallelotope $P=[-1,1]^{N_{1}}, X$ is closed and convex. However, it can be verified that $P$ has a countable dense subset while $X$ has not, and thus $X$ is not even a continuous image of $P$. (This example is due jointly to Dr. Ernest Michael and the author.)

A2. Convex sets and the nearest-point mapping. We next discuss the nearest-point mapping in normed linear spaces, since certain of its properties are needed in $\S I I 2$. If $X$ is a subset and $p$ a point of the metric space $(M, \rho)$, then $X p$ will denote the set (possibly empty) of all points $q \in X$ such that $\rho(p, q)=\inf _{x \in X} \rho(p, x) . X$ will be said to have the nearness property in $M$ iff $X p$ contains exactly one point for each $p \in M$. A subset $X$ of a normed linear

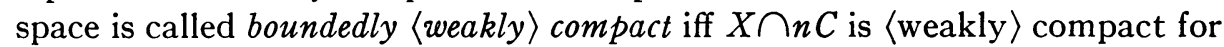
each $n, C$ being the unit cell. A convex set is boundedly /weakly) compact iff it is closed and locally 〈weakly > compact.

Standing hypotheses for this section: $E$ is a normed linear space and $K$ is a nonempty subset of $E$.

(A2.1) Suppose $E$ is strictly convex and $K$ is boundedly weakly compact. Then $K$ has the nearness property in $E$.

Proof. Consider an arbitrary $p \in E$. From the boundedly weak compactness of $K$ and the fact that $C$ is weakly closed it follows that there is at least one $t \geqq 0$ such that $(p+t C) \cap K \neq \Lambda$ and this intersection (for the least such $t$ ) is of course $K p$. That $K p$ contains only one point follows from the strict convexity of $E$.

We shall be concerned with spaces $E$ having the following property $(\mathrm{P})$ : for each pair $u, v$ of distinct points of $E$ and each $B>0$ there is a number $\delta>0$ and a weakly open set $W \ni u$ such that $\|x-u\|+\delta<\|x-v\|$ whenever $x \in W$ and $\|x\|<B$. It is easily verified that if $E$ is either finite-dimensional or an inner-product space, then $E$ has property (P).

(A2.2) Suppose $K$ is boundedly compact and has the nearness property in $E$. Then $K x \mid x \in E$ is continuous. If in addition $E$ has the property $\mathrm{P}$, then $K x \mid x \in E$ is weak-strong continuous.

Proof. Since the mapping $K$. takes bounded sets onto bounded sets, the first assertion follows easily from compactness and the triangle inequality. To prove the second it suffices to show that if $(A,>)$ is a directed set and $p a \mid a \in A$ is a bounded function on $A$ to $E$ such that (relative to $>$ ) $p a$ converges weakly to $p_{0} \in E$ and $K p a$ converges to $q$, then $q=K p_{0}$. Now suppose $K p_{0} \neq q$ and let $u=K p_{0}, v=q, B=\sup \{\|p a\| \| a \in A\}$. Let $\delta, W$ be as in the definition of property $(\mathrm{P})$. Then ultimately (in terms of $>$ ) we have $\left\|p a-K p_{0}\right\|+\delta<\|p a-q\|$. But since $K p a$ converges to $q$, ultimately $\|p a-q\|<\left\|p a-K p_{0}\right\|$, a contradiction completing the proof.

An easy application of the triangle inequality yields

(A2.3) Suppose $x \in E, q \in K x$, and $y \in[x, q]$. Then $q \in K y$. 
Our principal result on the nearness property is

(A2.4) Suppose $K$ has the nearness property in $E, x \in E \sim K, K x=y$, and $p \in \operatorname{Ray}[y, x[$. Then each of the following implies that $K p=y$.

(i) $E$ is finite-dimensional;

(ii) $E$ is reflexive and $K x \mid x \in E$ is continuous in both weak and strong topologies.

(iii) $K$ is convex and $E$ is strictly convex.

Proof. Since the nearness property implies that $K$ is closed, if $E$ is finitedimensional $K$ is boundedly compact and we see from (A2.2) that case (i) is included in (ii). Before proving (ii), let us deduce (iii) from (i). Let $x, y$, and $p$ be as in the hypotheses and suppose $K p=z \neq y$. Let $E^{\prime}$ be the linear hull of $\{\phi, x, y, p, z\}$ and $K^{\prime}=E^{\prime} \cap K$. Then $K^{\prime}$ is boundedly compact and convex, and $E^{\prime}$ is strictly convex, so it follows from (A2.1) that $K^{\prime}$ has the nearness property in $E^{\prime}$. But $K^{\prime} x=y$ and $K^{\prime} p=z \neq y$, which contradicts case (i) and completes the proof of (iii) (once case (ii) is successfully handled).

Turning now to case (ii), we note first that (due to (A2.3)) we need merely $K(y+t(x-y))=y$ for arbitrarily large values of $t$. Suppose this is not the case and take $y=\phi$. Then there is a point $z$ (a multiple of $x$ ) such that $K z=\phi$ but $K t z \neq \phi$ if $t>1$. By continuity of $K$. there are bounded closed convex sets $U$ and $V$ and a hyperplane $H \ni \phi$ such that $\phi \in \operatorname{Int} U, z \in \operatorname{Int} V, K V \subset U$, and $z / 2+H$ separates $U$ from $V$. By the choice of $z$ and the continuity of $\mathrm{K}$. there is a number $s>1$ and a bounded closed convex set $W^{\prime} \ni \phi$ such that $W^{\prime} \subset H \cap U, \phi \in$ Int $W^{\prime}$ (relative to $H$ ), $s z+W^{\prime} \subset V$, and $K\left(s z+W^{\prime}\right) \ni \phi$. But since $s z+W^{\prime}$ is weakly compact and $K$. weakly continuous, $K\left(s z+W^{\prime}\right)$ is weakly compact, hence closed and at positive distance from $\phi$. Thus for $W=r W^{\prime}$ and a sufficiently small $r>0$, there is no point $u \in s z+W$ such that $[u, K u]$ intersects $[s z, z]$.

For each $w \in W$ let $f w$ be defined by the condition, $z+f w \in[s z+W$, $K(s z+w)] \cap(z+H)$. Then $f$ is weakly continuous. Let $m>0$ be such that $m f w \in W$ and for each $t \in[0,1]$ and $w \in W$ let $g(t, w)=(1-t) w+t m f w$. Then each $g(t, \cdot)$ is a weakly continuous map of $W$ into itself. By the choice of $r$, the segment $[w, f w]$ contains $\phi$ only when $w=\phi$, and even then $f \phi \neq \phi$. Thus $\phi \in g(t, W)$ only when $t=0$.

Now by a proof analogous to that of $[10$, p. 75], using the Tychonoff fixed-point theorem [31] and the weak compactness of $W$, it can be shown that (in a sense involving both weak and strong topologies) $\phi$ is a stable value of the identity map $h$ on $W$. Specifically, there is an $\epsilon>0$ such that if $h^{\prime}$ is any weakly continuous map of $W$ onto itself for which $\left\|h^{\prime} w-h w\right\|<\epsilon$ for all $w \in W$, then $\phi \in h^{\prime} W$. But $h=g(0, \cdot)$ and $\lim _{t \rightarrow 0} \sup _{w \in W}\|g(t, w)-g(0, w)\|=0$, so we have a contradiction to the fact that $\phi \in g(t, W)$ only when $t=0$. This completes the proof of (A1.4).

From (A2.4) we deduce a well-known characterization of convexity and a generalization thereof. 
(A2.5) Suppose $E$ is smooth and strictly convex. Then each of the following additional conditions implies that $K$ is closed and convex iff $K$ has the nearness property in $E$ : (i) $E$ is finite-dimensional; (ii) $E$ is reflexive and has property $(\mathrm{P})$ and $K$ is boundedly compact.

Proof. In both cases the "only if" part follows from (A2.1). Suppose conversely that $K$ has the nearness property in $E$ and for each $x \in E \sim K$ let $Q_{x}$ be the (unique) closed half-space which contains $K x$ but does not intersect $N_{\|x-K x\|} x$. From (A1.4) and the smoothness of $E$ it follows that $K$ $\subset \cap_{x \in E \sim K} Q_{x}$. On the other hand, if $x \notin K$, then $x \notin Q_{x}$, so the reverse inclusion is also valid and $K$ is convex.

For a much simpler proof of case (i) of (A2.5), see [5] and [11].

In connection with the results of this section, the following result of Kuratowski [20] may be of interest: Suppose $X$ is a metrizable space and $A$ a subset of $X$. Then if $A$ is a retract of $X, X$ admits a metric for which $A$ has the nearness property in $X$.

\section{REFERENCES}

1. Stefan Banach, Théorie des opérations linéaires, Warsaw, 1932.

2. S. Banach and S. Mazur, Zur Theorie der linearen Dimension, Studia Math. vol. 4 (1933) pp. 100-112.

3. W. A. Blankinship, Generalization of a construction by Antoine, Ann. of Math. vol. 53 (1951) pp. 276-297.

4. K. Borsuk, Über Isomorphie der Funktionalraume, Bulletin International de l'Académie Polonaise des Sciences et des Lettres Ser. A (1933) pp. 1-10.

5. H. Busemann, Note on a theorem on convex sets, Matematisk Tidsskrift (1947) pp. 32-34.

6. J. A. Clarkson, Uniformly convex spaces, Trans. Amer. Math. Soc. vol. 40 (1936) pp. 396-414.

7. J. Dugundji, An extension of Tietze's theorem, Pacific Journal of Mathematics vol. 1 (1951) pp. 353-367.

8. W. F. Eberlein, Weak compactness in Banach spaces, I, Proc. Nat. Acad. Sci. U. S. A. vol. 33 (1947) pp. 51-53.

9. Samuel Eilenberg, On the problems of topology, Ann. of Math. vol. 50 (1949) pp. 247-260.

10. Witold Hurewicz and Henry Wallman, Dimension theory, Princeton, 1941.

11. B. Jessen, Two theorems on convex points sets (Danish), Matematisk Tidsskrift (1940) pp. 66-70.

12. S. Kaczmarz, The homeomorphy of certain spaces, Bulletin International de l'Académie Polonaise des Sciences et des Lettres Ser. A (1933) pp. 145-148.

13. Shizuo Kakutani, Topological properties of the unit sphere of a Hilbert space, Proc. Imp. Acad. Tokyo vol. 19 (1943) pp. 269-271.

14. Ott-Heinrich Keller, Die Homoiomorphie der kompakten konvexen Mengen im Hilbertschen Raum, Math. Ann. vol. 105 (1931) pp. 748-758.

15. V. L. Klee, Jr., Convex sets in linear spaces, Duke Math. J. vol. 18 (1951) pp. 443-466.

16. - Convex sets in linear spaces. II., Duke Math. J. vol. 18 (1951) pp. 875-883.

17. - Invariant metrics in groups (solution of a problem of Banach), Proceedings of the American Mathematical Society vol. 3 (1952) pp. 484-487.

18. M. Krein, D. Milman, and M. Rutman, $A$ note on basis in Banach space, Communications de l'Institut des Sciences Mathématiques et Mécaniques de l'Université de Kharkoff [et de la Société Mathématique de Kharkoff] (4) vol. 6 (1940) pp. 106-110. 
19. M. Krein and V. Smulian, On regularly convex sets in the space conjugate to a Banach space, Ann. of Math. (2) vol. 41 (1940) pp. 556-583.

20. Kazimierz Kuratowski, Une condition metrique pour la retraction des ensembles, Comptes Rendus des Séances de la Société des Sciences et des Lettres de Varsovie vol. 28 (1936) pp. 156158.

21. Solomon Lefschetz, Algebraic topology, Amer. Math. Soc. Colloquium Publications, vol. 27, New York, 1942.

22. S. Mazur, Une remarque sur l'homeomorphie des champs fonctionnels, Studia Math. vol. 1 (1929) pp. 83-85.

23. - Über die kleinsten konvexe Mengen, die eine gegebene kompakte Menge enthalt, Studia Math. vol. 2 (1930) pp. 7-9.

24. Edward James McShane, Integration, Princeton, 1944.

25. D. Montgomery, Pointwise periodic homeomorphisms, Amer. J. Math. vol. 59 (1937) pp. 118-120.

26. P. A. Smith, Fixed-point theorems for periodic transformations, Amer. J. Math. vol. 63 (1941) pp. 1-8.

27. - Transformations of finite period, III. Newman's theorem, Ann. of Math. vol. 42 (1941) pp. 446-458.

28. V. Smulian, On the principle of inclusion in the space of the type (B), Rec. Math. (Mat. Sbornik) N.S. vol. 5 (1939) pp. 317-328.

29. J. J. Stoker, Unbounded convex point sets, Amer. J. Math. vol. 62 (1940) pp. 165-179.

30. M. H. Stone, Notes on Integration. II, Proc. Nat. Acad. Sci. U. S. A. vol. 34 (1948) pp. 447-455.

31. A. Tychonoff, Ein Fixpunktsatz, Math. Ann. vol. 111 (1935) pp. 767-776.

UNIVER SITY OF VIRGINIA,

Charlottesville, Va.

The Institute for Advanced Study,

Princeton, N. J. 